\title{
THE EFFECTS OF HARVESTING AND TIME DELAY ON PREDATOR-PREY SYSTEMS WITH HOLLING TYPE II FUNCTIONAL RESPONSE*
}

\author{
JING XIA ${ }^{\dagger}$, ZHIHUA LIU ${ }^{\dagger}$, RONG YUAN ${ }^{\dagger}$, AND SHIGUI RUAN $\ddagger$
}

\begin{abstract}
In this paper, the effects of harvesting and time delay on two different types of predator-prey systems with delayed predator specific growth and Holling type II functional response are studied by applying the normal form theory of retarded functional differential equations developed by Faria and Magalhães [J. Differential Equations, 122 (1995), pp. 181-200, J. Differential Equations, 122 (1995), pp. 201-224]. Hopf bifurcations are demonstrated in models with harvesting of the prey at a constant rate by taking the delay as a bifurcation parameter, and numerical examples supporting our theoretical prediction are also given. Furthermore, bifurcation analysis indicates that delayed predator-prey systems with predator harvesting exhibit Bogdanov-Takens bifurcation. The versal unfoldings of the models at the Bogdanov-Takens singularity are obtained, and numerical simulations and bifurcation diagrams are given to illustrate the obtained results.
\end{abstract}

Key words. predator-prey system, harvesting, time delay, Hopf bifurcation, Bogdanov-Takens bifurcation

AMS subject classifications. 34K18, 37G05, 37G10, 92D25

DOI. $10.1137 / 080728512$

1. Introduction. The Canadian Grand Banks off the coast of Newfoundland were prime fishing grounds for several centuries. The population of Atlantic cod (Godus morhua) used to be so abundant that, in 1968, about 40,000 people were employed in the fishing industry and more than 800,000 tons of Atlantic cod were harvested (Schiermeier [34]). However, the Atlantic cod stocks collapsed in about 10 years and in 1992, the Department of Fisheries and Oceans of Canada belatedly closed the fisheries (Hutchings and Myers [21], Myers et al. [27, 28], Hutchings [20]). More than a decade later, there is still no sign of recovery of Atlantic cod stocks in the Grand Banks. Today, the fear of a rapid depletion of world fish stocks because of overexploitation is increasing (Pauly et al. [31], Myers and Worm [29]). Depletions of marine fish stocks not only endanger the future of marine fisheries but also may lead to species extinction and ecosystem regime shifts (Casey and Myers [6], Jackson et al. [22]). Thus, understanding the dynamics of fishing becomes very important.

There are several types of interactions within fisheries systems. Biological, harvest technical, and market interactions are the most important ones in bioeconomic modeling (Flaaten [16]). The biological interactions are predator-prey relations and competition between species. In the case of predator-prey interactions it is well known that the reduction of the predator stock level may increase the surplus production of the prey. However, harvesting becomes controversial when it comes to predators like whales and seals (May et al. [26], Flaaten [15], Yodzis [38]). The purpose of this paper is to study the importance of harvesting on multispecies management within

* Received by the editors June 25, 2008; accepted for publication (in revised form) June 22, 2009; published electronically September 18, 2009.

http://www.siam.org/journals/siap/70-4/72851.html

†School of Mathematical Sciences, Beijing Normal University, Beijing 100875, People's Republic of China (xiajing2005@mail.bnu.edu.cn, zhihualiu@bnu.edu.cn, ryuan@bnu.edu.cn). The research of these authors was partially supported by the National Nature Science Foundation of China.

${ }^{\ddagger}$ Department of Mathematics, University of Miami, Coral Gables, FL 33124-4250 (ruan@math. miami.edu). This author's research was partially supported by NSF grant DSM-0715772.

1178 
the framework of predator-prey models (Clark [8], Flaaten [16]).

The study of predator-prey models with harvesting has attracted the attention of many researchers. Let $x(t)$ and $y(t)$ denote the density of the prey and predators at time $t$, respectively. Consider constant-yield harvesting of a predator-prey system with Holling type II functional response:

$$
\left\{\begin{array}{l}
\dot{x}=r x\left(1-\frac{x}{K}\right)-\left(\frac{m}{\delta}\right) \frac{y x}{A+x}-H_{1}, \\
\dot{y}=y\left(-D+\frac{m x}{A+x}\right)-H_{2},
\end{array}\right.
$$

where $r$ is the intrinsic growth rate of the prey; $K$ is the carrying capacity of the prey; $m$ is the maximum growth rate of predators; $\delta$ is the yield conversion factor for predators feedings on the prey; $A$ is the half saturation constant for the predators which is the prey density at which the functional response is half maximal; $D$ is the death rate of predators; $H_{1}$ and $H_{2}$ are constant harvesting rates for the prey and predators, respectively. Note that we need to assume that $\dot{x} \geq 0$ and $\dot{y} \geq 0$ for all time. Model (1.1) and its variants have been studied extensively; see, for example, Brauer and Soudack [3, 4, 5], Beddington and Cooke [1], Dai and Tang [11], Hogarth et al. [19], Myerscough et al. [30], Xiao and Jennings [35], and Xiao and Ruan [36]. Very rich and interesting dynamical behaviors such as the existence of multiple equilibria, homoclinic loop, and Hopf bifurcation have been observed.

Let $\bar{y}=y / \delta, \bar{t}=m t, \bar{r}=r / m, \bar{H}_{1}=H_{1} / m, \bar{D}=D / m$, and $\bar{H}_{2}=H_{2} / m \delta$. Dropping the bars we obtain the dimensionless model

$$
\left\{\begin{array}{l}
\dot{x}=r x\left(1-\frac{x}{K}\right)-\frac{y x}{A+x}-H_{1}, \\
\dot{y}=y\left(-D+\frac{x}{A+x}\right)-H_{2} .
\end{array}\right.
$$

When $H_{1}=0$, Xiao and Ruan [36] carried out a bifurcation analysis of model (1.2). In particular they showed that codimension 2 bifurcations occur in a two-dimensional parameter region. Under some conditions they proved that system (1.2) undergoes Bogdanov-Takens bifurcation; i.e., it can exhibit qualitatively different dynamical behavior, including Hopf bifurcation, saddle-node bifurcation, as well as homoclinic bifurcation.

On the other hand, population models with time delay are of current research interest in mathematical biology because of their realistic meaning; we refer to the monographs of Cushing [10], Gopalsamy [17], and Kuang [23] for general delayed biological systems and a survey paper of Ruan [33] and the references cited therein for studies on delayed predator-prey systems. Brauer [2] was the first to consider the combined effects of time delay and constant harvesting on predator-prey models. Further studies were performed by Martin and Ruan [25] who studied the combined effects of the prey harvesting and time delay on the dynamics of the generalized Gausetype predator-prey models and the Wangersky-Cunningham model. It is shown that in these models the time delay can cause a stable equilibrium to become unstable and even a switching of stabilities, while the prey harvesting rate has a stabilizing effect on the equilibrium if it is under the critical harvesting level. In particular, one of these models loses stability when the delay varies and then regains its stability when the prey harvesting rate is increased.

In this paper, following the work of Martin and Ruan [25] and Xiao and Ruan [36], we continue studying how time delay and harvesting affect the dynamics of the 
predator-prey systems. We assume that a time delay $\tau(>0)$ occurs in the predator response term. It represents a gestation time of the predators. The reproduction of predators after predating the prey is not instantaneous but will be mediated by some discrete time lag required for gestation of the predators. We consider two cases.

Model 1. The prey population is harvested at a constant rate:

$$
\left\{\begin{array}{l}
\dot{x}(t)=r x(t)\left(1-\frac{x(t)}{K}\right)-\frac{x(t) y(t)}{A+x(t)}-H, \\
\dot{y}(t)=y(t)\left(-D+\frac{x(t-\tau)}{A+x(t-\tau)}\right) .
\end{array}\right.
$$

Model 2. The predator population is harvested at a constant rate:

$$
\left\{\begin{array}{l}
\dot{x}(t)=r x(t)\left(1-\frac{x(t)}{K}\right)-\frac{x(t) y(t)}{A+x(t)}, \\
\dot{y}(t)=y(t)\left(-D+\frac{x(t-\tau)}{A+x(t-\tau)}\right)-H .
\end{array}\right.
$$

The initial conditions for these systems are

$$
x(\theta)=\phi_{1}(\theta), \quad y(\theta)=\phi_{2}(\theta)
$$

for all $\theta \in[-\tau, 0]$, where $\left(\phi_{1}, \phi_{2}\right) \in C\left([-\tau, 0], \mathbb{R}_{+}^{2}\right), x(0)=\phi_{1}(0)>0$, and $y(0)=$ $\phi_{2}(0)>0$.

Following Xiao and Ruan [37], we first show that Hopf bifurcation occurs in Model 1 and determine the direction of the Hopf bifurcation. We then show that Model 2 exhibits Bogdanov-Takens bifurcation.

The paper is organized as follows. In section 2, we show that the system (1.3) with prey harvesting undergoes Hopf bifurcation as the delays cross some critical values and determine the stability of the bifurcating periodic solutions by using the method of Faria and Magalhães [13]. Numerical simulations are performed to illustrate the obtained results. In section 3, following the technique of Faria and Magalhães [13, 14], we show that the model (1.4) with predator harvesting exhibits Bogdanov-Takens bifurcation and obtain versal unfoldings at the Bogdanov-Takens singularity under some conditions. A brief discussion is given in section 4 to conclude the paper.

2. Prey harvesting. In this section, we are concerned with Model 1, i.e., system (1.3). We first study the interior equilibrium of system (1.3). An easy computation shows that when

$$
r A D(K-K D-A D)-H K(1-D)^{2}>0 \quad \text { and } \quad 0<D<1,
$$

system (1.3) has a unique positive equilibrium $E=\left(x_{0}, y_{0}\right)$, where $x_{0}=\frac{A D}{1-D}, y_{0}=$ $\left(r-\frac{r x_{0}}{K}-\frac{H}{x_{0}}\right)\left(A+x_{0}\right)$. Through transformation $z_{1}(t)=x(t)-x_{0}, z_{2}(t)=y(t)-y_{0}$, the equilibrium $E=\left(x_{0}, y_{0}\right)$ is translated to the origin $O=(0,0)$ and system (1.3) can be rewritten as the following equivalent system:

$$
\left\{\begin{array}{l}
\dot{z}_{1}(t)=\alpha_{1} z_{1}(t)+\alpha_{2} z_{2}(t)+\sum_{i+j \geq 2} \frac{1}{i ! j !} f_{i j}^{(1)} z_{1}^{i}(t) z_{2}^{j}(t), \\
\dot{z}_{2}(t)=\beta_{1} z_{1}(t-\tau)+\sum_{i+j \geq 2} \frac{1}{i ! j !} f_{i j}^{(2)} z_{1}^{i}(t-\tau) z_{2}^{j}(t),
\end{array}\right.
$$


where

$$
\begin{aligned}
\alpha_{1} & =r-\frac{2 x_{0} r}{K}-\frac{A y_{0}}{\left(A+x_{0}\right)^{2}}, \quad \alpha_{2}=-\frac{x_{0}}{A+x_{0}}, \quad \beta_{1}=\frac{A y_{0}}{\left(A+x_{0}\right)^{2}}, \\
f_{i j}^{(1)} & =\left.\frac{\partial^{i+j} f^{(1)}}{\partial x^{i} \partial y^{j}}\right|_{\left(x_{0}, y_{0}\right)}, \quad f_{i j}^{(2)}=\left.\frac{\partial^{i+j} f^{(2)}}{\partial x^{i} \partial y^{j}}\right|_{\left(x_{0}, y_{0}\right)}, \quad i, j \geq 0, \\
f^{(1)} & =r x\left(1-\frac{x}{K}\right)-\frac{x y}{A+x}-H, \quad f^{(2)}=y\left(-D+\frac{x}{A+x}\right) .
\end{aligned}
$$

We obtain the linearized system

$$
\left\{\begin{array}{l}
\dot{z}_{1}(t)=\alpha_{1} z_{1}(t)+\alpha_{2} z_{2}(t) \\
\dot{z}_{2}(t)=\beta_{1} z_{1}(t-\tau)
\end{array}\right.
$$

The characteristic equation of the linearized system (2.3) is a transcendental equation of the following form (see Cooke and Grossman [9] and Ruan [32]):

$$
\lambda^{2}-\lambda \alpha_{1}-\alpha_{2} \beta_{1} e^{-\lambda \tau}=0 .
$$

In [25], Martin and Ruan considered the following system:

$$
\left\{\begin{array}{l}
\dot{x}(t)=x(t)[f(x(t))-y(t) h(x(t))]-H \\
\dot{y}(t)=y(t)[-d+c x(t-\tau) h(x(t-\tau))] .
\end{array}\right.
$$

We note that when $f(x)$ is the logistic growth function and $x h(x)$ is the Holling type II response function in (2.5), then system (2.5) becomes system (1.3). Martin and Ruan studied the stability of the equilibria and existence of Hopf bifurcation for model (2.5). However, the direction of the Hopf bifurcation and stability of the bifurcating periodic solutions were not considered in [25]. In this section, we shall determine the direction and stability of the bifurcating periodic solutions by employing the normal form theory due to Faria and Magalhães [13].

For convenience, some useful lemmas and theorems from [25] are rewritten as follows.

Lemma 2.1. Assume that (2.1) holds and

$$
r K A D^{2}+(1-D)^{3} H K-r A^{2} D^{2}-r K A D^{3}-r A^{2} D^{3}<0 .
$$

Then at

$$
\tau_{k}=\frac{1}{\sigma_{+}}\left(\arccos \frac{-\sigma_{+}^{2}}{\alpha_{2} \beta_{1}}+2 k \pi\right), \quad k=0,1,2, \ldots,
$$

(2.4) has a simple pair of purely imaginary roots $\pm i \sigma_{+}$, where

$$
\sigma_{+}=\sqrt{\frac{-\alpha_{1}^{2}+\sqrt{\alpha_{1}^{4}+4 \alpha_{2}^{2} \beta_{1}^{2}}}{2}} .
$$

Furthermore,

(1) if $\tau \in\left[0, \tau_{0}\right)$, all roots of (2.4) have strictly negative real parts;

(2) if $\tau=\tau_{0}$, all roots of (2.4), except $\pm i \sigma_{+}$, have strictly negative real parts.

Copyright (C) by SIAM. Unauthorized reproduction of this article is prohibited. 
LEMMA 2.2. The following transversality condition

$$
\operatorname{sign}\left\{\operatorname{Re}\left(\frac{d \lambda}{d \tau}\right)\right\}_{\tau=\tau_{k}}>0
$$

is satisfied, where $\lambda(\tau)=\mu(\tau) \pm i \sigma(\tau)$ are the roots of $(2.4)$ near $\tau=\tau_{k}(k=0,1,2, \ldots)$ satisfying $\mu\left(\tau_{k}\right)=0, \sigma\left(\tau_{k}\right)=\sigma_{+}$, and $k=0,1,2, \ldots$

LEMMA 2.3. Suppose that (2.1) and (2.6) hold, then (2.4) has at least one eigenvalue with strictly positive real part for $\tau>\tau_{0}$.

THEOREM 2.4. Suppose that conditions (2.1) and (2.6) are satisfied. Then

(1) when $\tau \in\left[0, \tau_{0}\right)$, the zero solution of $(2.2)$ is locally asymptotically stable;

(2) when $\tau>\tau_{0}$, the zero solution of (2.2) is unstable;

(3) $\tau_{k}(k=0,1,2, \ldots)$ are Hopf bifurcation values for system (2.2).

From the above theorem, we know that system (1.3) undergoes Hopf bifurcations at the critical values $\tau_{k}(k=0,1,2, \ldots)$. In the following, we shall determine the direction and stability of the bifurcating periodic solutions.

Let $z_{1}(t)=x(\tau t)-x_{0}, z_{2}(t)=y(\tau t)-y_{0}$. Then system (1.3) is transformed into functional differential equations in $C\left([-1,0], \mathbb{R}^{2}\right)$

$$
\left\{\begin{array}{l}
\dot{z}_{1}(t)=\tau\left[\alpha_{1} z_{1}(t)+\alpha_{2} z_{2}(t)+\sum_{i+j \geq 2} \frac{1}{i ! j !} f_{i j}^{(1)} z_{1}^{i}(t) z_{2}^{j}(t)\right], \\
\dot{z}_{2}(t)=\tau\left[\beta_{1} z_{1}(t-1)+\sum_{i+j \geq 2} \frac{1}{i ! j} f_{i j}^{(2)} z_{1}^{i}(t-1) z_{2}^{j}(t)\right],
\end{array}\right.
$$

and the linearized system is

$$
\left\{\begin{array}{l}
\dot{z}_{1}(t)=\tau\left[\alpha_{1} z_{1}(t)+\alpha_{2} z_{2}(t)\right] \\
\dot{z}_{2}(t)=\tau \beta_{1} z_{1}(t-1)
\end{array}\right.
$$

System (2.10) has the characteristic equation

$$
\lambda^{2}-\lambda \tau \alpha_{1}-\tau^{2} \alpha_{2} \beta_{1} e^{-\lambda}=0 .
$$

Letting $\lambda=\xi \tau$, then (2.11) becomes

$$
\xi^{2}-\alpha_{1} \xi-\alpha_{2} \beta_{1} e^{-\xi \tau}=0 .
$$

Obviously, (2.12) is the same as (2.4). We know that for fixed $k \in N,(2.12)$ has a simple pair of conjugate complex roots $\xi(\tau)=\mu(\tau) \pm i \sigma(\tau)$ which satisfy

$$
\mu\left(\tau_{k}\right)=0, \quad \sigma\left(\tau_{k}\right)=\sigma_{+}, \quad \mu^{\prime}\left(\tau_{k}\right) \neq 0,
$$

where $\tau_{k}$ is given in (2.7). Therefore, the characteristic equation (2.11) has two complex roots $\lambda(\tau)=\tau \mu(\tau) \pm i \tau \sigma(\tau)$, which satisfy

$$
\left.\frac{d \operatorname{Re} \lambda(\tau)}{d \tau}\right|_{\tau=\tau_{k}}=\left.\tau \frac{d \operatorname{Re} \xi(\tau)}{d \tau}\right|_{\tau=\tau_{k}}+\left.\operatorname{Re} \xi(\tau)\right|_{\tau=\tau_{k}}=\tau_{k} \mu^{\prime}\left(\tau_{k}\right) \neq 0 .
$$

Let $z=\left(z_{1}, z_{2}\right)^{T}$. System (2.9) can further be written as

$$
\dot{z}(t)=L(\tau)\left(z_{t}\right)+F\left(z_{t}, \tau\right)
$$

Copyright (C) by SIAM. Unauthorized reproduction of this article is prohibited. 
where

$$
\begin{aligned}
L(\tau)(\varphi)=\tau\left(\begin{array}{c}
\alpha_{1} \varphi_{1}(0)+\alpha_{2} \varphi_{2}(0) \\
\beta_{1} \varphi_{1}(-1)
\end{array}\right), \\
F(\varphi, \tau)=\tau\left(\begin{array}{c}
\Sigma_{i+j \geq 2} \frac{1}{i ! j !} f_{i j}^{(1)} \varphi_{1}^{i}(0) \varphi_{2}^{j}(0) \\
\sum_{i+j \geq 2} \frac{1}{i ! j} f_{i j}^{(2)} \varphi_{1}^{i}(-1) \varphi_{2}^{j}(0)
\end{array}\right),
\end{aligned}
$$

where $\varphi=\left(\varphi_{1}, \varphi_{2}\right)^{T} \in C\left([-1,0], \mathbb{R}^{2}\right)$.

By the Riesz representation theorem, there exists an $n \times n$ matrix $\eta(\theta, \tau)$, whose elements are of bounded variation for $\theta \in[-1,0]$, such that for $\varphi \in C\left([-1,0], \mathbb{R}^{2}\right)$,

$$
L(\tau)(\varphi)=\int_{-1}^{0} d \eta(\theta, \tau) \varphi(\theta)
$$

Choose

$$
\eta(\theta, \tau)=\tau\left(\begin{array}{cc}
\alpha_{1} & \alpha_{2} \\
0 & 0
\end{array}\right) H(\theta)+\tau\left(\begin{array}{cc}
0 & 0 \\
-\beta_{1} & 0
\end{array}\right) H(\theta+1),
$$

where $H(\theta)$ is the Heaviside function. We expand $F(\varphi, \tau)$ about $\varphi$ as the Taylor expansion

$$
F(\varphi, \tau)=\frac{1}{2 !} F_{2}(\varphi, \tau)+\frac{1}{3 !} F_{3}(\varphi, \tau)+O\left(|\varphi|^{4}\right),
$$

where $F_{l}(\varphi, \tau)$ is given by

$$
\frac{1}{l !} F_{l}(\varphi, \tau)=\tau\left(\begin{array}{c}
\sum_{i+j=l} \frac{1}{i ! j !} f_{i j}^{(1)} \varphi_{1}^{i}(0) \varphi_{2}^{j}(0) \\
\sum_{i+j=l} \frac{1}{i ! j !} f_{i j}^{(2)} \varphi_{1}^{i}(-1) \varphi_{2}^{j}(0)
\end{array}\right)
$$

Setting a new parameter $\alpha=\tau-\tau_{k}$, system (2.13) is rewritten as

$$
\dot{z}(t)=L\left(\tau_{k}\right)\left(z_{t}\right)+F_{0}\left(z_{t}, \alpha\right),
$$

where $F_{0}(\varphi, \alpha)=L(\alpha)(\varphi)+F\left(\varphi, \tau_{k}+\alpha\right)$.

Let $A_{0}$ be the infinitesimal generator corresponding to $\dot{z}(t)=L\left(\tau_{k}\right) z_{t}$. Then $A_{0}$ has a pair of purely imaginary characteristic roots $\pm i \sigma_{k}\left(\sigma_{k}=\tau_{k} \sigma_{+}\right)$, which are simple, and no other characteristic roots with zero real part. Consider $\Lambda=\left\{-i \sigma_{k}, i \sigma_{k}\right\}$ and denote by $P$ the invariant space of $A_{0}$ corresponding to $\Lambda$, where $\operatorname{dim} P=2$. Let the phase space $C=C\left([-1,0], \mathbb{R}^{2}\right)$ be decomposed by $\Lambda$ as $C=P \oplus Q$ by applying the formal adjoint theory for functional differential equations in Hale [18]. Consider complex coordinates and still write as $C=C\left([-1,0], \mathbb{C}^{2}\right)$. Suppose that $\Phi=\left(\Phi_{1}, \Phi_{2}\right)$ is the basis for $P$ and $\Phi_{1}(\theta)=e^{i \sigma_{k} \theta} v^{T}=e^{i \sigma_{k} \theta}\left(1, v_{2}\right)^{T}, \Phi_{2}(\theta)=\overline{\Phi_{1}(\theta)},-1 \leq \theta \leq 0$, where $v=\left(1, v_{2}\right)^{T}$ is a vector in $\mathbb{C}^{2}$ and $v_{2}=\frac{\tau_{k} \beta_{1} e^{-i \sigma_{k}}}{i \sigma_{k}}$, which satisfies

$$
L\left(\tau_{k}\right)\left(\Phi_{1}\right)=i \sigma_{k} v^{T} .
$$

Copyright $@$ by SIAM. Unauthorized reproduction of this article is prohibited. 
Also, the two eigenvectors $\Psi_{1}$ and $\Psi_{2}$ of the formal adjoint operator $A_{0}^{*}$, corresponding to $i \sigma_{k}$ and $-i \sigma_{k}$, respectively, form a basis $\Psi(s)=\operatorname{col}\left(\Psi_{1}(s), \Psi_{2}(s)\right)$ of the adjoint space $P^{*}$ of $P$, where $\Psi_{1}(s)=e^{-i \sigma_{k} s}\left(u_{1}, u_{2}\right), \Psi_{2}(s)=\overline{\Psi_{1}(s)}, 0 \leq s \leq 1$. We know $-\dot{\Psi}_{1}(0)=\int_{-1}^{0} \Psi_{1}(-\theta) d \eta(\theta)$; therefore, we can choose $u_{2}=\frac{\tau_{k} \alpha_{2}}{i \sigma_{k}} u_{1}$. In order to have $(\Psi, \Phi)=\left(\left(\Psi_{j}, \Phi_{i}\right), i, j=1,2\right)=I_{2}$, where $I_{2}$ is the second-order identical matrix, $(\cdot ;)$ is a bilinear inner product form

$$
(\psi, \varphi)=\psi(0) \varphi(0)-\int_{-1}^{0} \int_{0}^{\theta} \psi(\xi-\theta) d \eta(\theta) \varphi(\xi) d \xi
$$

we have $u_{1}=\frac{\tau_{k}^{2} \beta_{1} \alpha_{2}}{\tau_{k}^{2} \beta_{1} \alpha_{2}+\left(i \sigma_{k}-\tau_{k} \alpha_{1}\right)^{2} e^{i \sigma_{k}}+\left(i \sigma_{k}-\tau_{k} \alpha_{1}\right) \tau_{k}^{2} \alpha_{2} \beta_{1}}$. Note that $\dot{\Phi}=\Phi B$, where $B$ is a diagonal matrix

$$
B=\left(\begin{array}{cc}
i \sigma_{k} & 0 \\
0 & i \sigma_{k}
\end{array}\right)
$$

Take the enlarged phase space $B C:=\left\{\varphi:[-1,0] \rightarrow \mathbb{C}^{2} \mid \varphi\right.$ is continuous on $[-1,0)$, $\left.\exists \lim _{\theta \rightarrow 0^{-}} \varphi(\theta)\right\}$. The elements of $B C$ have the form $\psi=\varphi+X_{0} \alpha$, where $\varphi \in C$, $\alpha \in \mathbb{C}^{2}, X_{0}=X_{0}(\theta)$ is given by

$$
X_{0}(\theta)= \begin{cases}I, & \theta=0 \\ 0, & -1 \leq \theta<0 .\end{cases}
$$

The projection of $C$ upon $P, \varphi \mapsto \Phi(\Psi, \varphi)$, associated with the decomposition $C=$ $P \oplus Q$ is replaced by $\pi: B C \mapsto P$ such that

$$
\pi\left(\varphi+X_{0} \alpha\right)=\Phi[(\Psi, \varphi)+\Psi(0) \alpha] .
$$

Thus, we have the decomposition $B C=P \oplus \operatorname{ker} \pi$. Use the decomposition $z_{t}=$ $\Phi x(t)+y_{t}, x(t) \in \mathbb{C}^{2}, y_{t} \in \operatorname{ker} \pi \cap C^{1} \triangleq Q^{1}$, where $C^{1}=C^{1}\left([-1,0] ; \mathbb{R}^{2}\right)$ denotes the space of continuously differentiable functions from $[-1,0]$ to $\mathbb{R}^{2}$. The equation $(2.16)$ is equivalent to the system

$$
\left\{\begin{array}{l}
\dot{x}=B x+\Psi(0) F_{0}(\Phi x+y, \alpha), \\
\frac{d}{d t} y=A_{Q^{1}} y+(I-\pi) X_{0} F_{0}(\Phi x+y, \alpha) .
\end{array}\right.
$$

We have the Taylor expansions,

$$
\begin{gathered}
\Psi(0) F_{0}(\Phi x+y, \alpha)=\frac{1}{2} f_{2}^{1}(x, y, \alpha)+\frac{1}{3 !} f_{3}^{1}(x, y, \alpha)+\text { h.o.t. } \\
(I-\pi) X_{0} F_{0}(\Phi x+y, \alpha)=\frac{1}{2} f_{2}^{2}(x, y, \alpha)+\frac{1}{3 !} f_{3}^{2}(x, y, \alpha)+\text { h.o.t. }
\end{gathered}
$$

where $f_{j}^{1}(x, y, \alpha)$ and $f_{j}^{2}(x, y, \alpha)$ are homogeneous polynomials in $(x, y, \alpha)$ of degree $j, j=2,3$, with coefficients in $\mathbb{C}^{2}$ and $\operatorname{ker} \pi$, respectively, and h.o.t. stands for higher order terms. The normal form method implies a normal form on the center manifold of the origin for $(2.17)$ as

$$
\dot{x}=B x+\frac{1}{2} g_{2}^{1}(x, 0, \alpha)+\frac{1}{3 !} g_{3}^{1}(x, 0, \alpha)+\text { h.o.t },
$$

Copyright $@$ by SIAM. Unauthorized reproduction of this article is prohibited. 
where $g_{2}^{1}, g_{3}^{1}$ are the second- and third-order terms in $(x, \alpha)$, respectively. Following [13], $V_{j}^{m+p}(X)$ denotes the linear space of homogeneous polynomials of degree $j$ in $m+p$ real variables, $x=\left(x_{1}, \ldots, x_{m}\right), \alpha=\left(\alpha_{1}, \ldots, \alpha_{p}\right)$ with coefficients in $X$; that is,

$$
V_{j}^{m+p}(X)=\left\{\sum_{|(q, l)|=j} c_{(q, l)} x^{q} \alpha^{l}:(q, l) \in N_{0}^{m+p}, c_{(q, l)} \in X\right\} .
$$

The operators $M_{j}^{1}$ are defined by

$$
\left(M_{j}^{1} p\right)(x, \alpha)=D_{x} p(x, \alpha) B x-B p(x, \alpha), \quad j \geq 2,
$$

and $M_{j}^{1}$ act in $V_{j}^{3}\left(\mathbb{C}^{2}\right), V_{j}^{3}\left(\mathbb{C}^{2}\right)=\operatorname{Im}\left(M_{j}^{1}\right) \oplus \operatorname{ker}\left(M_{j}^{1}\right)$ and

$$
\operatorname{ker}\left(M_{j}^{1}\right)=\operatorname{span}\left\{x^{q} \alpha^{l} e_{k}:(q, \bar{\lambda})=\lambda_{k}, k=1,2, q \in \mathbb{N}_{0}^{2}, l \in \mathbb{N}_{0},|(q, l)|=j\right\},
$$

where $\left\{e_{1}, e_{2}\right\}$ is the canonical basis of $\mathbb{C}^{2}$. Hence,

$$
\begin{aligned}
& \operatorname{ker}\left(M_{2}^{1}\right)=\operatorname{span}\left\{\left(\begin{array}{c}
x_{1} \alpha \\
0
\end{array}\right),\left(\begin{array}{c}
0 \\
x_{2} \alpha
\end{array}\right)\right\}, \\
& \operatorname{ker}\left(M_{3}^{1}\right)=\operatorname{span}\left\{\left(\begin{array}{c}
x_{1}^{2} x_{2} \\
0
\end{array}\right),\left(\begin{array}{c}
x_{1} \alpha^{2} \\
0
\end{array}\right),\left(\begin{array}{c}
0 \\
x_{1} x_{2}^{2}
\end{array}\right),\left(\begin{array}{c}
0 \\
x_{2} \alpha^{2}
\end{array}\right)\right\} .
\end{aligned}
$$

For (2.18), it follows that

$$
f_{2}^{1}(x, y, \alpha)=\Psi(0)\left[2 L(\alpha)(\Phi x+y)+F_{2}\left(\Phi x+y, \tau_{k}\right)\right],
$$

where $F_{2}$ is given in (2.15). By computing, we obtain

$$
f_{2}^{1}(x, 0, \alpha)=\left(\begin{array}{c}
2 A_{1} x_{1} \alpha+2 A_{2} x_{2} \alpha+a_{20} x_{1}^{2}+2 a_{11} x_{1} x_{2}+a_{02} x_{2}^{2} \\
2 \bar{A}_{2} x_{1} \alpha+2 \bar{A}_{1} x_{2} \alpha+\bar{a}_{02} x_{1}^{2}+2 \bar{a}_{11} x_{1} x_{2}+\bar{a}_{20} x_{2}^{2}
\end{array}\right),
$$

where

$$
\begin{aligned}
A_{1} & =\frac{i \sigma_{k}}{\tau_{k}}\left(u_{1}+u_{2} v_{2}\right), \\
A_{2} & =\frac{-i \sigma_{k}}{\tau_{k}}\left(u_{1}+u_{2} \bar{v}_{2}\right), \\
a_{20} & =\tau_{k}\left[u_{1} f_{20}^{(1)}+2 u_{1} v_{2} f_{11}^{(1)}+u_{2} f_{20}^{(2)} e^{-2 i \sigma_{k}}+2 u_{2} v_{2} f_{11}^{(2)} e^{-i \sigma_{k}}\right], \\
a_{11} & =\tau_{k}\left[u_{1} f_{20}^{(1)}+u_{1} f_{11}^{(1)}\left(v_{2}+\bar{v}_{2}\right)+u_{2} f_{20}^{(2)}+u_{2} f_{11}^{(2)}\left(e^{-i \sigma_{k}} \bar{v}_{2}+e^{i \sigma_{k}} v_{2}\right)\right], \\
a_{02} & =\tau_{k}\left[u_{1} f_{20}^{(1)}+2 u_{1} \bar{v}_{2} f_{11}^{(1)}+u_{2} f_{20}^{(2)} e^{2 i \sigma_{k}}+2 u_{2} \bar{v}_{2} f_{11}^{(2)} e^{i \sigma_{k}}\right] .
\end{aligned}
$$

Since the second-order term in $(\alpha, x)$ of the normal form on the center manifold is given by

$$
\frac{1}{2} g_{2}^{1}(x, 0, \alpha)=\frac{1}{2} \operatorname{Proj}_{\operatorname{ker}\left(M_{2}^{1}\right)} f_{2}^{1}(x, 0, \alpha)
$$

it implies that

$$
\frac{1}{2} g_{2}^{1}(x, 0, \alpha)=\left(\begin{array}{c}
A_{1} x_{1} \alpha \\
\bar{A}_{1} x_{2} \alpha
\end{array}\right)
$$

Copyright $@$ by SIAM. Unauthorized reproduction of this article is prohibited. 
where $A_{1}=\frac{i \sigma_{k}}{\tau_{k}}\left(u_{1}+u_{2} v_{2}\right)$. Note that

$$
g_{3}^{1}(x, 0, \alpha) \in \operatorname{ker}\left(M_{3}^{1}\right)=\operatorname{span}\left\{\left(\begin{array}{c}
x_{1}^{2} x_{2} \\
0
\end{array}\right),\left(\begin{array}{c}
x_{1} \alpha^{2} \\
0
\end{array}\right),\left(\begin{array}{c}
0 \\
x_{1} x_{2}^{2}
\end{array}\right),\left(\begin{array}{c}
0 \\
x_{2} \alpha^{2}
\end{array}\right)\right\} .
$$

However, the terms $O\left(|x| \alpha^{2}\right)$ are irrelevant to determine the generic Hopf bifurcation. Hence, we need only to compute the coefficients of $\left(\begin{array}{c}x_{1}^{2} x_{2} \\ 0\end{array}\right)$ and $\left(\begin{array}{c}0 \\ x_{1} x_{2}^{2}\end{array}\right)$. Notice that

$$
\frac{1}{3 !} g_{3}^{1}(x, 0, \alpha)=\frac{1}{3 !} \operatorname{Proj}_{\operatorname{ker}\left(M_{3}^{1}\right)} \tilde{f}_{3}^{1}(x, 0, \alpha)=\frac{1}{3 !} \operatorname{Proj}_{s} \tilde{f}_{3}^{1}(x, 0,0)+O\left(|x| \alpha^{2}\right),
$$

where

$$
s:=\operatorname{span}\left\{\left(\begin{array}{c}
x_{1}^{2} x_{2} \\
0
\end{array}\right),\left(\begin{array}{c}
0 \\
x_{1} x_{2}^{2}
\end{array}\right)\right\}
$$

and the term $\tilde{f}_{3}^{1}(x, 0,0)$ is given by

$$
\tilde{f}_{3}^{1}(x, 0,0)=f_{3}^{1}(x, 0,0)+\frac{3}{2}\left[\left(D_{x} f_{2}^{1}\right) U_{2}^{1}-\left(D_{x} U_{2}^{1}\right) g_{2}^{1}\right]_{(x, 0,0)}+\frac{3}{2}\left[\left(D_{y} f_{2}^{1}\right) h\right]_{(x, 0,0)} .
$$

Now we compute $\frac{1}{3 !} g_{3}^{1}(x, 0, \alpha)$ step by step.

Step 1. We compute $\operatorname{Proj}_{s}\left[\left(D_{x} f_{2}^{1}\right) U_{2}^{1}\right]_{(x, 0,0)}$. Following [13], we take

$$
U_{2}^{1}(x, 0)=\left(M_{2}^{1}\right)^{-1} P_{I, 2}^{1} f_{2}^{1}(x, 0,0) .
$$

We have from (2.21) that

$$
f_{2}^{1}(x, 0,0)=\left(\begin{array}{c}
a_{20} x_{1}^{2}+2 a_{11} x_{1} x_{2}+a_{02} x_{2}^{2} \\
\bar{a}_{02} x_{1}^{2}+2 \bar{a}_{11} x_{1} x_{2}+\bar{a}_{20} x_{2}^{2}
\end{array}\right) .
$$

According to the definition of $M_{2}^{1}$, the equation $M_{2}^{1} U_{2}^{1}(x, 0)=f_{2}^{1}(x, 0,0)$ can be written as the following differential equations:

$$
\left\{\begin{array}{l}
x_{1} \frac{\partial u_{1}}{\partial x_{1}}-x_{2} \frac{\partial u_{1}}{\partial x_{2}}-u_{1}=\frac{1}{i \sigma_{k}}\left(a_{20} x_{1}^{2}+2 a_{11} x_{1} x_{2}+a_{02} x_{2}^{2}\right), \\
x_{1} \frac{\partial u_{2}}{\partial x_{1}}-x_{2} \frac{\partial u_{2}}{\partial x_{2}}+u_{2}=\frac{1}{i \sigma_{k}}\left(\bar{a}_{02} x_{1}^{2}+2 \bar{a}_{11} x_{1} x_{2}+\bar{a}_{20} x_{2}^{2}\right) .
\end{array}\right.
$$

A straightforward calculation shows that

$$
U_{2}^{1}(x, 0)=\left(\begin{array}{c}
\frac{1}{i \sigma_{k}}\left(a_{20} x_{1}^{2}-2 a_{11} x_{1} x_{2}-\frac{1}{3} a_{02} x_{2}^{2}\right) \\
\frac{1}{i \sigma_{k}}\left(\frac{1}{3} \bar{a}_{02} x_{1}^{2}+2 \bar{a}_{11} x_{1} x_{2}-\bar{a}_{20} x_{2}^{2}\right)
\end{array}\right) .
$$

Consequently,

$$
\operatorname{Proj}_{s}\left[\left(D_{x} f_{2}^{1}\right) U_{2}^{1}\right]_{(x, 0,0)}=\left(\begin{array}{c}
\frac{2}{i \sigma_{k}}\left(\frac{1}{3}\left|a_{02}\right|^{2}+2\left|a_{11}\right|^{2}-a_{11} a_{20}\right) x_{1}^{2} x_{2} \\
\frac{-2}{i \sigma_{k}}\left(\frac{1}{3}\left|a_{02}\right|^{2}+2\left|a_{11}\right|^{2}-\bar{a}_{11} \bar{a}_{20}\right) x_{1} x_{2}^{2}
\end{array}\right) .
$$

Copyright $@$ by SIAM. Unauthorized reproduction of this article is prohibited. 
Step 2. We compute $\operatorname{Proj}_{s}\left[\left(D_{x} U_{2}^{1}\right) g_{2}^{1}\right]_{(x, 0,0)}$. From (2.23), we know that $g_{2}^{1}(x, 0,0)$ $=0$. Thus, $\left[\left(D_{x} U_{2}^{1}\right) g_{2}^{1}\right]_{(x, 0,0)}=0$.

Step 3. We compute $\operatorname{Proj}_{s}\left[\left(D_{y} f_{2}^{1}\right) h\right]_{(x, 0,0)}$, where $h=\left(h^{1}, h^{2}\right)^{T}$ is a second degree homogeneous polynomial in $\left(x_{1}, x_{2}, \alpha\right)$ with coefficients in $Q^{1}$, which has the form

$$
h=h\left(x_{1}, x_{2}, \alpha\right)=h_{110} x_{1} x_{2}+h_{101} x_{1} \alpha+h_{011} x_{2} \alpha+h_{200} x_{1}^{2}+h_{020} x_{2}^{2}+h_{002} \alpha^{2},
$$

and $h=h\left(x_{1}, x_{2}, \alpha\right)$ is the unique solution in $V_{2}^{3}\left(Q^{1}\right)$ of the equation

$$
\left(M_{2}^{2} h\right)(x, \alpha)=(I-\pi) X_{0}\left[2 L(\alpha)(\Phi x)+F_{2}\left(\Phi x, \tau_{k}\right)\right] .
$$

Since

$$
\begin{aligned}
\left(M_{2}^{2} h\right)(x, \alpha) & =D_{x} h(x, \alpha) B x-A_{Q^{1}}(h(x, \alpha)) \\
& =D_{x} h(x, \alpha) B x-\dot{h}(x, \alpha)-X_{0}\left[L\left(\tau_{k}\right)(h(x, \alpha))-\dot{h}(x, \alpha)(0)\right] \\
& =(I-\pi) X_{0}\left[2 L(\alpha)(\Phi x)+F_{2}\left(\Phi x, \tau_{k}\right)\right] ;
\end{aligned}
$$

thus, $h=h(x, 0)(\theta)$ can be evaluated by the system

$$
\begin{aligned}
& \dot{h}(x)-D_{x} h(x) B x=\Phi \Psi(0) F_{2}\left(\Phi x, \tau_{k}\right), \\
& \dot{h}(x)(0)-L\left(\tau_{k}\right)(h(x))=F_{2}\left(\Phi x, \tau_{k}\right),
\end{aligned}
$$

where $\dot{h}$ denotes the derivative of $h(x)(\theta)$ with respect to $\theta$. From (2.20), we obtain

$$
\begin{aligned}
f_{2}^{1}(x, y, 0) & =\Psi(0) F_{2}\left(\Phi x+y, \tau_{k}\right) \\
& =\left(\begin{array}{cc}
u_{1} & u_{2} \\
\bar{u}_{1} & \bar{u}_{2}
\end{array}\right) \tau_{k}\left(\begin{array}{c}
f_{20}^{(1)} p_{1}^{2}+2 f_{11}^{(1)} p_{1} p_{2} \\
f_{20}^{(2)} l_{1}^{2}+2 f_{11}^{(2)} l_{1} p_{2}
\end{array}\right),
\end{aligned}
$$

where

$$
p_{1}=x_{1}+x_{2}+y_{1}(0), \quad p_{2}=v_{2} x_{1}+\bar{v}_{2} x_{2}+y_{2}(0), \quad l_{1}=e^{-i \sigma_{k}} x_{1}+e^{i \sigma_{k}} x_{2}+y_{1}(-1) .
$$

Hence, we have

$$
\left[\left(D_{y} f_{2}^{1}\right) h\right]_{(x, 0,0)}=\tau_{k}\left(\begin{array}{c}
u^{T}\left(\begin{array}{c}
2 f_{20}^{(1)} p_{1}^{\prime} h^{1}(0)+2 f_{11}^{(1)} p_{2}^{\prime} h^{1}(0)+2 f_{11}^{(1)} p_{1}^{\prime} h^{2}(0) \\
2 f_{20}^{(2)} l_{1}^{\prime} h^{1}(-1)+2 f_{11}^{(2)} p_{2}^{\prime} h^{1}(0)+2 f_{11}^{(2)} l_{1}^{\prime} h^{2}(-1)
\end{array}\right) \\
\bar{u}^{T}\left(\begin{array}{c}
2 f_{20}^{(1)} p_{1}^{\prime} h^{1}(0)+2 f_{11}^{(1)} p_{2}^{\prime} h^{1}(0)+2 f_{11}^{(1)} p_{1}^{\prime} h^{2}(0) \\
2 f_{20}^{(2)} l_{1}^{\prime} h^{1}(-1)+2 f_{11}^{(2)} p_{2}^{\prime} h^{1}(0)+2 f_{11}^{(2)} l_{1}^{\prime} h^{2}(-1)
\end{array}\right)
\end{array}\right),
$$

where

$$
p_{1}^{\prime}=x_{1}+x_{2}, \quad p_{2}^{\prime}=v_{2} x_{1}+\bar{v}_{2} x_{2}, \quad l_{1}^{\prime}=e^{-i \sigma_{k}} x_{1}+e^{i \sigma_{k}} x_{2} .
$$

Thus,

$$
\operatorname{Proj}_{s}\left[\left(D_{y} f_{2}^{1}\right) h\right]_{(x, 0,0)}=\left(\begin{array}{c}
2 c_{3} x_{1}^{2} x_{2} \\
2 \bar{c}_{3} x_{1} x_{2}^{2}
\end{array}\right)
$$

where

$c_{3}=u^{T} \tau_{k}\left(\begin{array}{c}a h_{110}^{1}(0)+\bar{a} h_{200}^{1}(0)+b h_{110}^{2}(0)+\bar{b} h_{200}^{2}(0) \\ c h_{110}^{1}(-1)+\bar{c} h_{200}^{1}(-1)+d h_{110}^{2}(-1)+\bar{d} h_{200}^{2}(-1)+e h_{110}^{1}(0)+\bar{e} h_{200}^{1}(0)\end{array}\right)$ 
and

$$
a=f_{20}^{(1)}+f_{11}^{(1)} v_{2}, \quad b=f_{11}^{(1)}, \quad c=f_{20}^{(2)} e^{-i \sigma_{k}}, \quad d=f_{11}^{(2)} e^{-i \sigma_{k}}, \quad e=f_{11}^{(2)} v_{2} .
$$

In the following, we compute $h_{110}(\theta)$ and $h_{200}(\theta)$. Combining (2.24) with (2.25), we know that $h_{110}=\left(h_{110}^{1}, h_{110}^{2}\right)^{T}$ and $h_{200}=\left(h_{200}^{1}, h_{200}^{2}\right)^{T}$ are, respectively, the solution of the following two systems:

$$
\left\{\begin{array}{l}
\dot{h_{110}}=\left(\begin{array}{ll}
\Phi_{1} & \Phi_{2}
\end{array}\right)\left(\begin{array}{l}
2 a_{11} \\
2 \bar{a}_{11}
\end{array}\right) \\
\dot{h_{110}(0)-L\left(\tau_{k}\right)\left(h_{110}\right)=\tau_{k}}\left(\begin{array}{l}
a_{1} \\
b_{1}
\end{array}\right)
\end{array}\right.
$$

and

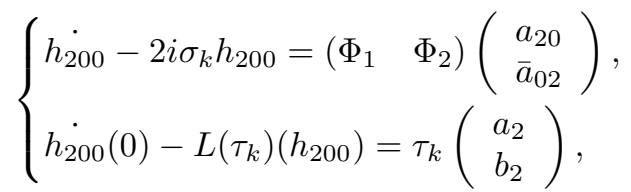

where

$$
\begin{aligned}
& a_{1}=2 f_{20}^{(1)}+2\left(v_{2}+\bar{v}_{2}\right) f_{11}^{(1)}, \quad b_{1}=2 f_{20}^{(2)}+2 f_{11}^{(2)}\left(e^{-i \sigma_{k}} \bar{v}_{2}+e^{i \sigma_{k}} v_{2}\right), \\
& a_{2}=f_{20}^{(1)}+2 f_{11}^{(1)} v_{2}, \quad b_{2}=f_{20}^{(2)} e^{-2 i \sigma_{k}}+2 f_{11}^{(2)} v_{2} e^{-i \sigma_{k}} .
\end{aligned}
$$

Solving (2.27) and (2.28), we obtain

$$
h_{110}(\theta)=\frac{2}{i \sigma_{k}}\left(a_{11} e^{i \sigma_{k} \theta} v-\bar{a}_{11} e^{-i \sigma_{k} \theta} \bar{v}\right)+c_{1}
$$

and

$$
h_{200}(\theta)=-\frac{1}{i \sigma_{k}}\left(a_{20} e^{i \sigma_{k} \theta} v+\frac{1}{3} \bar{a}_{02} e^{-i \sigma_{k} \theta} \bar{v}\right)+e^{2 i \sigma_{k} \theta} c_{2},
$$

where $c_{1}=\left(c_{1}^{(1)}, c_{1}^{(2)}\right)^{T}, c_{2}=\left(c_{2}^{(1)}, c_{2}^{(2)}\right)^{T}$, and

$$
\begin{aligned}
& c_{1}^{(1)}=-\frac{b_{1}}{\beta_{1}}, \quad c_{1}^{(2)}=\frac{\alpha_{1} b_{1}-\beta_{1} a_{1}}{\alpha_{2} \beta_{1}}, \\
& c_{2}^{(1)}=\frac{2 i \sigma_{k} \tau_{k} a_{2}+\tau_{k}^{2} \alpha_{2} b_{2}}{2 i \sigma_{k}\left(2 i \sigma_{k}-\tau_{k} \alpha_{1}\right)-\tau_{k}^{2} \alpha_{2} \beta_{1} e^{-2 i \sigma_{k}}}, \\
& c_{2}^{(2)}=\frac{\tau_{k}^{2} a_{2} \beta_{1} e^{-2 i \sigma_{k}}+2 i \sigma_{k} \tau_{k} b_{2}-\tau_{k}^{2} \alpha_{1} b_{2}}{2 i \sigma_{k}\left(2 i \sigma_{k}-\tau_{k} \alpha_{1}\right)-\tau_{k}^{2} \alpha_{2} \beta_{1} e^{-2 i \sigma_{k}}} .
\end{aligned}
$$

Step 4. We compute $\operatorname{Proj}_{s} f_{3}^{1}(x, 0,0)$. From $(2.18), f_{3}^{1}(x, 0,0)$ is given by

$$
f_{3}^{1}(x, 0,0)=\Psi(0) F_{3}\left(\Phi x, \tau_{k}\right),
$$

where $F_{3}\left(\Phi x, \tau_{k}\right)$ is defined in (2.15). Thus, $F_{3}$ can be computed as follows:

$$
F_{3}\left(\Phi x, \tau_{k}\right)=\tau_{k}\left(\begin{array}{c}
f_{30}^{(1)} p_{1}^{\prime 3}+3 f_{21}^{(1)} p_{1}^{\prime 2} p_{2}^{\prime} \\
f_{30}^{(2)} l_{1}^{\prime 3}+3 f_{21}^{(2)} l_{1}^{\prime 2} p_{2}^{\prime}
\end{array}\right),
$$

Copyright (c) by SIAM. Unauthorized reproduction of this article is prohibited. 
where $p_{1}^{\prime}, p_{2}^{\prime}, l_{1}^{\prime}$ are given in (2.26). Therefore, we obtain

$$
\operatorname{Proj}_{s} f_{3}^{1}(x, 0,0)=\left(\begin{array}{c}
3 a_{21} x_{1}^{2} x_{2} \\
3 \bar{a}_{21} x_{1} x_{2}^{2}
\end{array}\right),
$$

where

$$
a_{21}=\tau_{k} u_{1}\left(f_{30}^{(1)}+f_{21}^{(1)} \bar{v}_{2}+2 f_{21}^{(1)} v_{2}\right)+\tau_{k} u_{2}\left(f_{30}^{(2)} e^{-i \sigma_{k}}+f_{21}^{(2)} e^{-2 i \sigma_{k}} \bar{v}_{2}+2 f_{21}^{(2)} v_{2}\right) .
$$

Summarizing Steps 1-4, we obtain

$$
\frac{1}{3 !} g_{3}^{1}(x, 0,0)=\left(\begin{array}{c}
A_{3} x_{1}^{2} x_{2} \\
\bar{A}_{3} x_{1} x_{2}^{2}
\end{array}\right),
$$

where

$$
A_{3}=\frac{1}{2 i \sigma_{k}}\left(\frac{1}{3}\left|a_{02}\right|^{2}+2\left|a_{11}\right|^{2}-a_{11} a_{20}\right)+\frac{1}{2}\left(a_{21}+c_{3}\right) .
$$

In consequence, the normal form of (2.19) has the form

$$
\begin{aligned}
\dot{x} & =B x+\frac{1}{2} g_{2}^{1}(x, 0, \alpha)+\frac{1}{3 !} g_{3}^{1}(x, 0, \alpha)+\text { h.o.t. } \\
& =B x+\left(\begin{array}{c}
A_{1} x_{1} \alpha \\
\bar{A}_{1} x_{2} \alpha
\end{array}\right)+\left(\begin{array}{c}
A_{3} x_{1}^{2} x_{2} \\
\bar{A}_{3} x_{1} x_{2}^{2}
\end{array}\right)+O\left(|x| \alpha^{2}+|x|^{4}\right) .
\end{aligned}
$$

The normal form (2.19) relative to $P$ can be written in real coordinates $\left(w_{1}, w_{2}\right)$ through the change of variables $x_{1}=w_{1}-i w_{2}, x_{2}=w_{1}+i w_{2}$. Followed by the use of polar coordinates $(\rho, \xi), w_{1}=\rho \cos \xi, w_{2}=\rho \sin \xi$, this formal form becomes

$$
\left\{\begin{array}{l}
\dot{\rho}=k_{1} \alpha \rho+k_{2} \rho^{3}+O\left(\alpha^{2} \rho+|(\rho, \alpha)|^{4}\right) \\
\dot{\xi}=-\sigma_{k}+O(|(\rho, \alpha)|)
\end{array}\right.
$$

where $k_{1}=\operatorname{Re} A_{1}, \quad k_{2}=\operatorname{Re} A_{3}$. Following [7], we know that the sign of $k_{1} k_{2}$ determines the direction of the bifurcation and the sign of $k_{2}$ determines the stability of the nontrivial periodic solution bifurcating from Hopf bifurcation. Therefore, summarizing the above discussion, we have the following theorem.

THEOREM 2.5. The flow of (2.16) on the center manifold of the origin at $\tau=\tau_{k}$ is given by (2.32). Hopf bifurcation is supercritical if $k_{1} k_{2}<0$ and subcritical if $k_{1} k_{2}>0$. Moreover, the nontrivial periodic solution is stable if $k_{2}<0$ and unstable if $k_{2}>0$.

As an example, we consider system (1.3) with $A=1, D=\frac{2}{3}, H=\frac{1}{20}, K=4$, and $r=\frac{1}{2}$; that is,

$$
\left\{\begin{array}{l}
\dot{x}=\frac{1}{2} x\left(1-\frac{x}{4}\right)-\frac{x y}{1+x}-\frac{1}{20}, \\
\dot{y}=y\left(-\frac{2}{3}+\frac{x(t-\tau)}{1+x(t-\tau)}\right) .
\end{array}\right.
$$

In this case, system (2.33) has a unique positive equilibrium $\left(x_{0}, y_{0}\right)=\left(2, \frac{27}{40}\right)$ and conditions (2.1) and (2.6) are satisfied. Thus, the equilibrium $\left(x_{0}, y_{0}\right)$ is asymptotically stable when $\tau \in\left[0, \tau_{0}\right)$, where $\tau_{0}=1.5279$. By means of the software Maple, we 

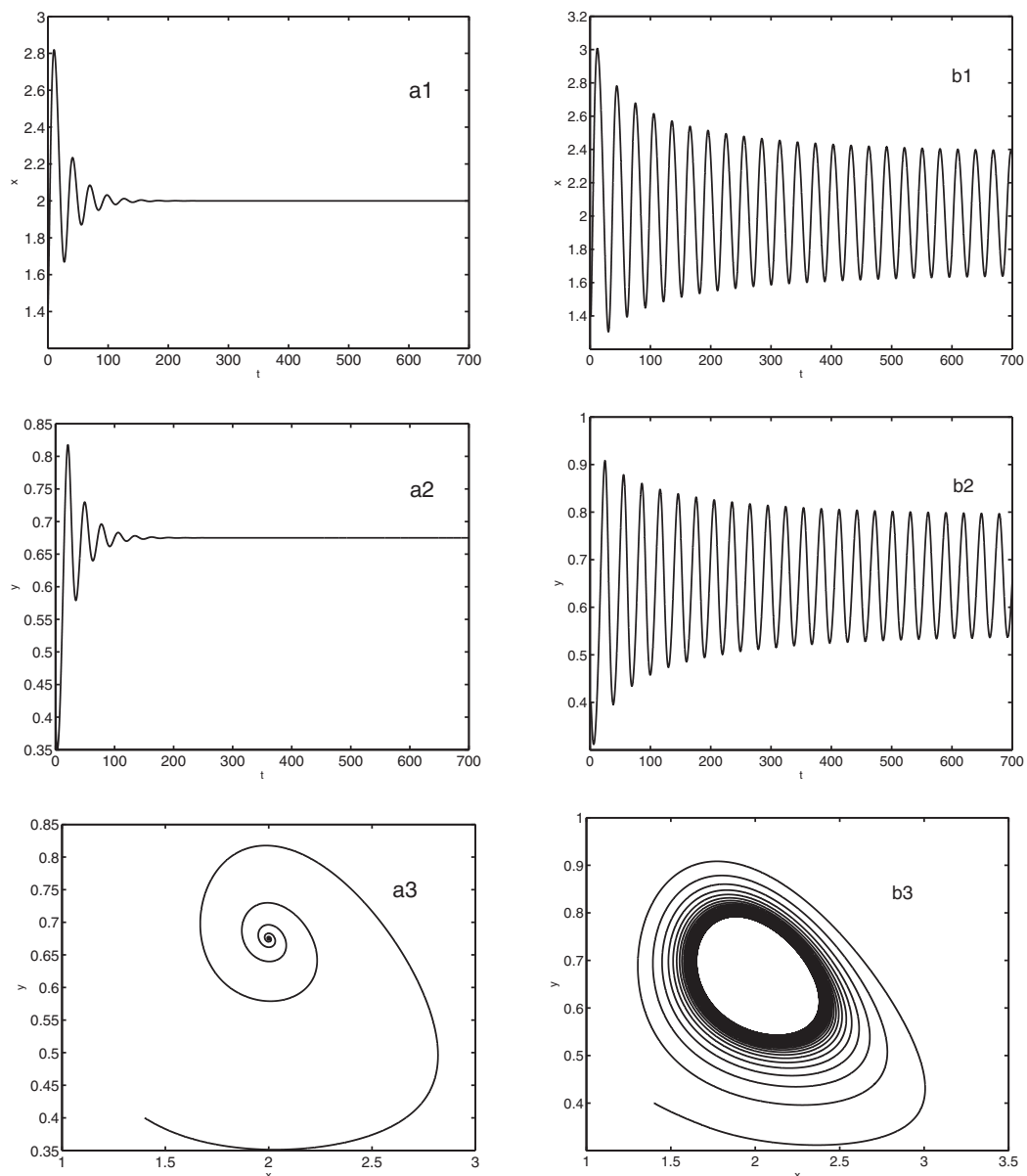

FIG. 1. The solution trajectories and phase portraits of the system (2.33) before (a1-a3) and after (b1-b3) Hopf bifurcation. In (a1)-(a3), $\tau=1.45$; in (b1)-(b3), $\tau=1.6$.

can compute the following values:

$$
v=\left(\begin{array}{l}
v_{1} \\
v_{2}
\end{array}\right)=\left(\begin{array}{c}
1 \\
-0.1125-0.3261 i
\end{array}\right), u=\left(\begin{array}{l}
u_{1} \\
u_{2}
\end{array}\right)=\left(\begin{array}{c}
0.4729+0.0029 i \\
-0.0088+1.4501 i
\end{array}\right)
$$

and

$$
\begin{aligned}
a_{20} & =-0.0602-0.1399 i, \quad a_{11}=-0.1258-0.1115 i, \\
a_{02} & =-0.1904-0.2455 i, \quad a_{21}=-0.0722+0.1053 i, \\
c_{3} & =0.1306+0.1172 i .
\end{aligned}
$$

Therefore, from (2.22) and (2.31), we can compute

$$
A_{1}=0.0342+0.2058 i, \quad A_{3}=-0.0074-0.0343 i .
$$

Thus, $k_{1}=0.0342, k_{2}=-0.0074$. By Theorem 2.5, we know that there is a supercritical Hopf bifurcation for (2.33) at $\tau=\tau_{0}$ and the nontrivial periodic solution associated with Hopf bifurcation is stable. The numerical simulations are depicted in Figure 1. 
Remark 2.6. Martin and Ruan [25] studied the Hopf bifurcation in the following predator-prey system with prey harvesting and delayed prey specific growth:

$$
\left\{\begin{array}{l}
\dot{x}(t)=r x(t)\left(1-\frac{x(t-\tau)}{K}\right)-\frac{x(t) y(t)}{A+x(t)}-H, \\
\dot{y}(t)=y(t)\left(-D+\frac{x(t)}{A+x(t)}\right) .
\end{array}\right.
$$

Similarly, as in Theorem 2.5 we can determine the direction of the Hopf bifurcation in system (2.34).

3. Predator harvesting. Following Xiao and Ruan [36], we know that system (1.4) has a unique interior equilibrium $E=\left(x_{0}, y_{0}\right)$ provided that

$$
\left(1-D+\frac{A D}{K}\right)^{2}-4 \frac{(1-D)(A D r+H)}{K r}=0 \quad \text { and } \quad K>\frac{A D}{1-D}
$$

hold and $\left(x_{0}, y_{0}\right)$ is given by $x_{0}=\frac{K(1-D)+A D}{2(1-D)}$ and $y_{0}=r\left(1-\frac{x_{0}}{K}\right)\left(A+x_{0}\right)$. We assume throughout this section that $0<D<1$.

We translate the equilibrium $\left(x_{0}, y_{0}\right)$ of system (1.4) to the origin. Setting $z_{1}(t)=$ $x(t)-x_{0}, z_{2}(t)=y(t)-y_{0}$, system (1.4) can be written as the following system:

$$
\left\{\begin{array}{l}
\dot{z}_{1}(t)=\alpha_{1} z_{1}(t)+\alpha_{2} z_{2}(t)+\sum_{i+j \geq 2} \frac{1}{i ! j !} f_{i j}^{(1)} z_{1}^{i}(t) z_{2}^{j}(t), \\
\dot{z}_{2}(t)=\beta_{1} z_{1}(t-\tau)+\beta_{2} z_{2}(t)+\sum_{i+j \geq 2} \frac{1}{i ! j !} f_{i j}^{(2)} z_{1}^{i}(t-\tau) z_{2}^{j}(t),
\end{array}\right.
$$

where

$$
\begin{aligned}
\alpha_{1} & =r-\frac{2 x_{0} r}{K}-\frac{A y_{0}}{\left(A+x_{0}\right)^{2}}, \quad \alpha_{2}=-\frac{x_{0}}{A+x_{0}}, \\
\beta_{1} & =\frac{A y_{0}}{\left(A+x_{0}\right)^{2}}, \quad \beta_{2}=-D+\frac{x_{0}}{A+x_{0}}, \\
f_{i j}^{(1)} & =\left.\frac{\partial^{i+j} f^{(1)}}{\partial x^{i} \partial y^{j}}\right|_{\left(x_{0}, y_{0}\right)}, \quad f_{i j}^{(2)}=\left.\frac{\partial^{i+j} f^{(2)}}{\partial x^{i} \partial y^{j}}\right|_{\left(x_{0}, y_{0}\right)}, \quad i, j \geq 0, \\
f^{(1)} & =r x\left(1-\frac{x}{K}\right)-\frac{x y}{A+x}, \quad f^{(2)}=y\left(-D+\frac{x}{A+x}\right)-H .
\end{aligned}
$$

Consider the linearized system of (3.2) at the zero equilibrium

$$
\left\{\begin{array}{l}
\dot{z}_{1}(t)=\alpha_{1} z_{1}(t)+\alpha_{2} z_{2}(t) \\
\dot{z}_{2}(t)=\beta_{1} z_{1}(t-\tau)+\beta_{2} z_{2}(t)
\end{array}\right.
$$

The characteristic equation for system (3.4) takes the form

$$
\lambda^{2}-\lambda\left(\alpha_{1}+\beta_{2}\right)+\alpha_{1} \beta_{2}-\alpha_{2} \beta_{1} e^{-\lambda \tau}=0 .
$$

In fact, we have $\alpha_{1} \beta_{2}-\alpha_{2} \beta_{1}=0$ (the proof can be found in the Appendix). We can easily see that (3.5) has two zero eigenvalues and no other eigenvalues on the imaginary axis if and only if $\tau=\tau_{0}=\frac{\alpha_{1}+\beta_{2}}{\alpha_{2} \beta_{1}}$ and $\tau_{0} \neq \sqrt{\frac{2}{\alpha_{2} \beta_{1}}}$. Because $\tau_{0}>0$ and $\alpha_{2} \beta_{1}<0$, we know that $\tau_{0} \neq \sqrt{\frac{2}{\alpha_{2} \beta_{1}}}$. 
Normalizing the delay $\tau$ in system (1.4) by the time-scaling $t \rightarrow t / \tau$, system (1.4) is transformed into

$$
\left\{\begin{array}{l}
\dot{x}(t)=\tau\left[r x(t)\left(1-\frac{x(t)}{K}\right)-\frac{x(t) y(t)}{A+x(t)}\right] \\
\dot{y}(t)=\tau y(t)\left[-D+\frac{x(t-1)}{A+x(t-1)}\right]-\tau H
\end{array}\right.
$$

Setting $z_{1}(t)=x(t)-x_{0}, z_{2}(t)=y(t)-y_{0}$, system (3.6) can be rewritten as functional differential equations in $C:=C\left([-1,0], \mathbb{R}^{2}\right)$

$$
\left\{\begin{array}{l}
\dot{z}_{1}(t)=\tau\left[\alpha_{1} z_{1}(t)+\alpha_{2} z_{2}(t)+\sum_{i+j \geq 2} \frac{1}{i ! j !} f_{i j}^{(1)} z_{1}^{i}(t) z_{2}^{j}(t)\right] \\
\dot{z}_{2}(t)=\tau\left[\beta_{1} z_{1}(t-1)+\beta_{2} z_{2}(t)+\sum_{i+j \geq 2} \frac{1}{i ! j !} f_{i j}^{(2)} z_{1}^{i}(t-1) z_{2}^{j}(t)\right] .
\end{array}\right.
$$

Let $z=\left(z_{1}, z_{2}\right)^{T}$. System (3.7) is written as

$$
\dot{z}(t)=\tau L\left(z_{t}\right)+\tau F\left(z_{t}\right)
$$

where $L: C \rightarrow \mathbb{R}^{2}, F: C \rightarrow \mathbb{R}^{2}$ are given by

$$
L(\varphi)=\left(\begin{array}{c}
\alpha_{1} \varphi_{1}(0)+\alpha_{2} \varphi_{2}(0) \\
\beta_{1} \varphi_{1}(-1)+\beta_{2} \varphi_{2}(0)
\end{array}\right), \quad F(\varphi)=\left(\begin{array}{c}
\sum_{i+j \geq 2} \frac{1}{i ! j !} f_{i j}^{(1)} \varphi_{1}^{i}(0) \varphi_{2}^{j}(0) \\
\sum_{i+j \geq 2} \frac{1}{i ! j !} f_{i j}^{(2)} \varphi_{1}^{i}(-1) \varphi_{2}^{j}(0)
\end{array}\right)
$$

and $\varphi=\operatorname{col}\left(\varphi_{1}, \varphi_{2}\right)$. We consider the formal Taylor expansion of $F$

$$
F(\varphi)=\sum_{j \geq 2} \frac{1}{j !} F_{j}(\varphi), \quad \varphi \in C
$$

and define $L_{0}=\tau_{0} L . L_{0}(\varphi)$ is a continuous linear function. Therefore, there exists a $2 \times 2$ matrix function $\eta(\theta),-1 \leq \theta \leq 0$, whose elements are of bounded variation such that

$$
L_{0}(\varphi)=\int_{-1}^{0} d \eta(\theta) \varphi(\theta)
$$

Choose

$$
\eta(\theta)=\tau_{0}\left(\begin{array}{cc}
\alpha_{1} & \alpha_{2} \\
0 & \beta_{2}
\end{array}\right) H(\theta)+\tau_{0}\left(\begin{array}{cc}
0 & 0 \\
-\beta_{1} & 0
\end{array}\right) H(\theta+1) .
$$

Let $A_{0}$ be the infinitesimal generator corresponding to $\dot{z}(t)=L_{0}\left(z_{t}\right)$. Then $A_{0}$ has two zero characteristic roots. Define $\Lambda=\{0\}$ and denote by $P$ the invariant space of $A_{0}$ corresponding to $\Lambda$, where the dimension of $P$ equals to 2 . Let $\Phi=\left(\Phi_{1}, \Phi_{2}\right)$ and $\Psi=\operatorname{col}\left(\Psi_{1}, \Psi_{2}\right)$ be the bases for $P$ and $P^{*}$, the adjoint space of $P$, respectively. We note that $\dot{\Phi}=\Phi B$, thus, for $\Phi=\left(\Phi_{1}, \Phi_{2}\right)$, and the matrix $B$ is given by

$$
B=\left(\begin{array}{ll}
0 & 1 \\
0 & 0
\end{array}\right) \text {. }
$$

Copyright $@$ by SIAM. Unauthorized reproduction of this article is prohibited. 
We know that $\Phi_{i}(0), \Psi_{i}(0)(i=1,2)$ satisfy $\dot{\Phi}_{i}(0)=\int_{-1}^{0} d \eta(\theta) \Phi_{i}(\theta),-\dot{\Psi}_{i}(0)=$ $\int_{-1}^{0} \Psi_{i}(-\theta) d \eta(\theta)$, and $(\Psi, \Phi)=\left(\left(\Psi_{j}, \Phi_{i}\right), i, j=1,2\right)=I_{2}$. Accordingly, we can obtain

$$
\begin{gathered}
\Phi(\theta)=\left(\begin{array}{cc}
-\alpha_{2} & -\alpha_{2} \theta-\alpha_{2} \\
\alpha_{1} & \alpha_{1} \theta+\alpha_{1}-\tau_{0}^{-1}
\end{array}\right), \\
\Psi(s)=\left(\begin{array}{cc}
-e s+g & -f s+h \\
e & f
\end{array}\right), \quad-1 \leq \theta \leq 0, \quad 0 \leq s \leq 1,
\end{gathered}
$$

where $e, f, g, h$ satisfy the following equations:

$$
\left\{\begin{array}{l}
\alpha_{2} e+\beta_{2} f=0 \\
\tau_{0} \alpha_{2} g+\tau_{0} \beta_{2} h=f \\
h\left(\alpha_{1}-\tau_{0} \alpha_{2} \beta_{1}\right)-\alpha_{2} g+\frac{1}{2} \tau_{0} \alpha_{2} \beta_{1} f=1 \\
h\left(\alpha_{1}-\tau_{0}^{-1}-\frac{1}{2} \tau_{0} \beta_{1} \alpha_{2}\right)-\alpha_{2} g+\frac{1}{3} \tau_{0} \beta_{1} \alpha_{2} f=0 \\
-e \alpha_{2}+f\left(\alpha_{1}-\tau_{0}^{-1}-\frac{1}{2} \tau_{0} \beta_{1} \alpha_{2}\right)=1 .
\end{array}\right.
$$

According to [14], we obtain that the normal form for (3.8) is as follows

$$
\left\{\begin{array}{l}
\dot{x}_{1}=x_{2}+O\left(\left|\left(x_{1}, x_{2}\right)\right|^{3}\right), \\
\dot{x}_{2}=B_{1} x_{1}^{2}+B_{2} x_{1} x_{2}+O\left(\left|\left(x_{1}, x_{2}\right)\right|^{3}\right),
\end{array}\right.
$$

where

$$
\begin{gathered}
B_{1}=\frac{1}{2} \tau \alpha_{2}^{2}\left(e f_{20}^{(1)}+f f_{20}^{(2)}\right)-\tau \alpha_{1} \alpha_{2}\left(e f_{11}^{(1)}+f f_{11}^{(2)}\right), \\
B_{2}=\tau \alpha_{2}^{2}\left(g f_{20}^{(1)}+h f_{20}^{(2)}+e f_{20}^{(1)}\right)-\tau \alpha_{1} \alpha_{2}\left(2 g f_{11}^{(1)}+2 e f_{11}^{(1)}\right. \\
\left.+2 h f_{11}^{(2)}+f f_{11}^{(2)}\right)+\tau \tau_{0}^{-1} \alpha_{2}\left(e f_{11}^{(1)}+f f_{11}^{(2)}\right),
\end{gathered}
$$

and $\alpha_{i}, f_{i j}^{(1)}, f_{i j}^{(2)}(i, j=1,2)$ are given in (3.3). In fact, the Bogdanov-Takens bifurcation for a planar system with two discrete delays have been studied in Faria [12]. Applying the formula in [12], we can also derive the above normal form. Therefore, we have the following result.

Theorem 3.1. Suppose that (3.1) holds. Then the equilibrium $E$ of (1.4) is a Bogdanov-Takens singularity for $\tau=\tau_{0}=\frac{\alpha_{1}+\beta_{2}}{\alpha_{2} \beta_{1}}$.

We know that system (1.4) undergoes Bogdanov-Takens bifurcation as $\tau$ crosses the critical value $\tau_{0}$. Next, we are interested in determining a versal unfolding for system (1.4) with a Bogdanov-Takens singularity. Note that $\alpha_{1} \beta_{2}-\alpha_{2} \beta_{1}=0$ (see the Appendix). We introduce two bifurcation parameters $\mu=\left(\mu_{1}, \mu_{2}\right)$ by setting $\tau=\tau_{0}+\mu_{1}, \beta_{1}=\frac{\alpha_{1} \beta_{2}}{\alpha_{2}}+\mu_{2}$. System (3.8) is rewritten as

$$
\dot{z}(t)=L_{0}\left(z_{t}\right)+L_{1}(\mu) z_{t}+\tilde{F}\left(z_{t}, \mu\right),
$$

where

$$
L_{1}(\mu) \varphi=\mu_{1} L(\varphi)+\tau_{0} \mu_{2}\left(\begin{array}{c}
0 \\
\varphi_{1}(-1)
\end{array}\right)
$$

Copyright (c) by SIAM. Unauthorized reproduction of this article is prohibited. 


$$
\tilde{F}(\varphi, \mu)=\mu_{1} \mu_{2}\left(\begin{array}{c}
0 \\
\varphi_{1}(-1)
\end{array}\right)+\left(\tau_{0}+\mu_{1}\right) F(\varphi) \text { for } \quad \varphi=\left(\begin{array}{c}
\varphi_{1} \\
\varphi_{2}
\end{array}\right) .
$$

In the phase space $B C=P \oplus \operatorname{ker} \pi$, we decompose $z_{t}$ in the form $z_{t}=\Phi x+y$. Thus, the system (3.12) is equivalent to

$$
\left\{\begin{array}{l}
\dot{x}=B x+\Psi(0)\left[L_{1}(\mu)(\Phi x+y)+\tilde{F}(\Phi x+y, \mu)\right], \\
\frac{d}{d t} y=A_{Q^{1}} y+(I-\pi) X_{0}\left[L_{1}(\mu)(\Phi x+y)+\tilde{F}(\Phi x+y, \mu)\right],
\end{array}\right.
$$

where $L_{1}(\mu) \varphi, \tilde{F}(\varphi, \mu)$ are given by (3.13) and (3.14) and $\varphi=\operatorname{col}\left(\varphi_{1}, \varphi_{2}\right)$. Define

$$
\begin{aligned}
& \Psi(0)\left[L_{1}(\mu)(\Phi x+y)+\tilde{F}(\Phi x+y, \mu)\right]=\frac{1}{2} f_{2}^{1}(x, y, \mu)+\frac{1}{3 !} f_{3}^{1}(x, y, \mu)+\text { h.o.t } \\
& (I-\pi) X_{0}\left[L_{1}(\mu)(\Phi x+y)+\tilde{F}(\Phi x+y, \mu)\right]=\frac{1}{2} f_{2}^{2}(x, y, \mu)+\frac{1}{3 !} f_{3}^{2}(x, y, \mu)+\text { h.o.t.. }
\end{aligned}
$$

Therefore, system (3.15) becomes

$$
\left\{\begin{array}{l}
\dot{x}=B x+\frac{1}{2} f_{2}^{1}(x, y, \mu)+\text { h.o.t. } \\
\frac{d}{d t} y=A_{Q^{1}} y+\frac{1}{2} f_{2}^{2}(x, y, \mu)+\text { h.o.t. }
\end{array}\right.
$$

where $f_{2}^{1}(x, y, \mu)$ and $f_{2}^{2}(x, y, \mu)$ are homogeneous polynomials in $(x, y, \mu)$ of degree 2 , with coefficients in $\mathbb{R}^{2}$ and $\operatorname{ker} \pi$, respectively. Thus, the normal form for (3.12) on the center manifold of the origin has the form

$$
\dot{x}=B x+\frac{1}{2} g_{2}^{1}(x, 0, \mu)+\text { h.o.t. }
$$

where $g_{2}^{1}$ is the second-order term in $x, \mu$ and determined by

$$
\frac{1}{2} g_{2}^{1}(x, 0, \mu)=\frac{1}{2} \operatorname{Proj}_{\operatorname{Im}\left(M_{2}^{1}\right)^{c}} f_{2}^{1}(x, 0, \mu),
$$

where $\operatorname{Im}\left(M_{2}^{1}\right)^{c}$ is a complementary space of $\operatorname{Im}\left(M_{2}^{1}\right)$ in $V_{2}^{4}\left(\mathbb{R}^{2}\right)$ and $V_{2}^{4}\left(\mathbb{R}^{2}\right)$ is the space of homogeneous polynomials of degree 2 in the variables $\left(x_{1}, x_{2}, \mu_{1}, \mu_{2}\right)$. According to (3.13), (3.14), and (3.16), we know that

$$
\begin{aligned}
f_{2}^{1}(x, 0, \mu) & =\Psi(0)\left[2 L_{1}(\mu)(\Phi x)+\tau_{0} F_{2}(\Phi x)\right] \\
& =\Psi(0)\left[\left(\begin{array}{c}
-2 \alpha_{2} \tau_{0}^{-1} \mu_{1} x_{2} \\
2 \beta_{2}\left(\alpha_{1}-\tau_{0}^{-1}\right) \mu_{1} x_{2}-2 \tau_{0} \alpha_{2} \mu_{2} x_{1}
\end{array}\right)+\tau_{0} F_{2}(\Phi x)\right],
\end{aligned}
$$

where $F_{2}$ is given in (3.9) and (3.10). We consider the canonical basis of $V_{2}^{4}\left(\mathbb{R}^{2}\right)$

$$
\begin{gathered}
\left(\begin{array}{c}
x_{1}^{2} \\
0
\end{array}\right),\left(\begin{array}{c}
x_{1} x_{2} \\
0
\end{array}\right),\left(\begin{array}{c}
x_{2}^{2} \\
0
\end{array}\right),\left(\begin{array}{c}
x_{1} \mu_{i} \\
0
\end{array}\right),\left(\begin{array}{c}
x_{2} \mu_{i} \\
0
\end{array}\right),\left(\begin{array}{c}
\mu_{1}^{2} \\
0
\end{array}\right),\left(\begin{array}{c}
\mu_{1} \mu_{2} \\
0
\end{array}\right),\left(\begin{array}{c}
\mu_{2}^{2} \\
0
\end{array}\right), \\
\left(\begin{array}{c}
0 \\
x_{1}^{2}
\end{array}\right),\left(\begin{array}{c}
0 \\
x_{1} x_{2}
\end{array}\right),\left(\begin{array}{c}
0 \\
x_{2}^{2}
\end{array}\right),\left(\begin{array}{c}
0 \\
x_{1} \mu_{i}
\end{array}\right),\left(\begin{array}{c}
0 \\
x_{2} \mu_{i}
\end{array}\right),\left(\begin{array}{c}
0 \\
\mu_{1}^{2}
\end{array}\right),\left(\begin{array}{c}
0 \\
\mu_{1} \mu_{2}
\end{array}\right) \\
\left(\begin{array}{c}
0 \\
\mu_{2}^{2}
\end{array}\right),(i=1,2) .
\end{gathered}
$$

Copyright $@$ by SIAM. Unauthorized reproduction of this article is prohibited. 
The operators $M_{j}^{1}$ are defined by

$$
\left(M_{j}^{1} p\right)(x)=D_{x} p(x) B x-B p(x), \quad j \geq 2 ;
$$

thus, $M_{2}^{1}$ acting in $V_{2}^{4}\left(\mathbb{R}^{2}\right)$ has the following form

$$
M_{2}^{1} p\left(x_{1}, x_{2}\right)=\left(\begin{array}{c}
x_{2} \frac{\partial p_{1}}{\partial x_{1}}-p_{2} \\
x_{2} \frac{\partial p_{2}}{\partial x_{1}}
\end{array}\right),
$$

where $p \in V_{2}^{4}\left(\mathbb{R}^{2}\right)$. The images of the basis under $M_{2}^{1}$ are, respectively,

$$
\begin{aligned}
& \left(\begin{array}{c}
2 x_{1} x_{2} \\
0
\end{array}\right),\left(\begin{array}{c}
x_{2}^{2} \\
0
\end{array}\right),\left(\begin{array}{l}
0 \\
0
\end{array}\right),\left(\begin{array}{c}
x_{2} \mu_{i} \\
0
\end{array}\right),\left(\begin{array}{l}
0 \\
0
\end{array}\right),\left(\begin{array}{l}
0 \\
0
\end{array}\right),\left(\begin{array}{c}
0 \\
0
\end{array}\right), \\
& \left(\begin{array}{c}
0 \\
0
\end{array}\right),\left(\begin{array}{c}
-x_{1}^{2} \\
2 x_{1} x_{2}
\end{array}\right),\left(\begin{array}{c}
-x_{1} x_{2} \\
x_{2}^{2}
\end{array}\right),\left(\begin{array}{c}
-x_{2}^{2} \\
0
\end{array}\right),\left(\begin{array}{c}
-x_{1} \mu_{i} \\
x_{2} \mu_{i}
\end{array}\right),\left(\begin{array}{c}
-x_{2} \mu_{i} \\
0
\end{array}\right), \\
& \left(\begin{array}{c}
-\mu_{1}^{2} \\
0
\end{array}\right),\left(\begin{array}{c}
-\mu_{1} \mu_{2} \\
0
\end{array}\right),\left(\begin{array}{c}
-\mu_{2}^{2} \\
0
\end{array}\right), \quad(i=1,2) .
\end{aligned}
$$

A complementary space of $\operatorname{Im}\left(M_{2}^{1}\right)$ in $V_{2}^{4}\left(\mathbb{R}^{2}\right)$ is

$$
\begin{gathered}
\operatorname{Im}\left(M_{2}^{1}\right)^{c}=\operatorname{span}\left\{\left(\begin{array}{c}
0 \\
x_{1}^{2}
\end{array}\right),\left(\begin{array}{c}
0 \\
x_{1} x_{2}
\end{array}\right),\left(\begin{array}{c}
0 \\
x_{1} \mu_{1}
\end{array}\right),\left(\begin{array}{c}
0 \\
x_{1} \mu_{2}
\end{array}\right),\left(\begin{array}{c}
0 \\
x_{2} \mu_{1}
\end{array}\right),\right. \\
\left.\left(\begin{array}{c}
0 \\
x_{2} \mu_{2}
\end{array}\right),\left(\begin{array}{c}
0 \\
\mu_{1}^{2}
\end{array}\right),\left(\begin{array}{c}
0 \\
\mu_{1} \mu_{2}
\end{array}\right),\left(\begin{array}{c}
0 \\
\mu_{2}^{2}
\end{array}\right)\right\} .
\end{gathered}
$$

Following [12], we know that the normal form of (3.12) on the center manifold is given by

$$
\left\{\begin{array}{l}
\dot{x}_{1}=x_{2}+\text { h.o.t. } \\
\dot{x}_{2}=\lambda_{1} x_{1}+\lambda_{2} x_{2}+B_{1} x_{1}^{2}+B_{2} x_{1} x_{2}+\text { h.o.t. }
\end{array}\right.
$$

where

$$
\lambda_{1}=-\tau_{0} \alpha_{2} f \mu_{2}, \quad \lambda_{2}=\beta_{2} \alpha_{1} f \mu_{1}-\tau_{0} \alpha_{2} h \mu_{2}
$$

and

$$
\begin{aligned}
B_{1} & =\frac{\tau_{0}}{2}\left[\alpha_{2}^{2}\left(e f_{20}^{(1)}+f f_{20}^{(2)}\right)-2 \alpha_{1} \alpha_{2}\left(e f_{11}^{(1)}+f f_{11}^{(2)}\right)\right], \\
B_{2} & =\tau_{0}\left\{\alpha_{2}^{2}\left(g f_{20}^{(1)}+h f_{20}^{(2)}\right)-2 \alpha_{1} \alpha_{2}\left(g f_{11}^{(1)}+h f_{11}^{(2)}\right)+\alpha_{2}^{2} e f_{20}^{(1)}\right. \\
& \left.-\alpha_{2}\left[\left(2 \alpha_{1}-\tau_{0}^{-1}\right) f_{11}^{(1)} e+\left(\alpha_{1}-\tau_{0}^{-1}\right) f_{11}^{(2)} f\right]\right\} .
\end{aligned}
$$

The above arguments imply the following theorem.

Theorem 3.2. Let $\mu_{1}, \mu_{2}$ be defined by $\tau=\tau_{0}+\mu_{1}, \beta_{1}=\frac{\alpha_{1} \beta_{2}}{\alpha_{2}}+\mu_{2}$, where $\tau_{0}=\frac{\alpha_{1}+\beta_{2}}{\alpha_{2} \beta_{1}}$. For $E=\left(x_{0}, y_{0}\right)$ and $\mu_{1}=0, \mu_{2}=0$, system (1.4) exhibits a BogdanovTakens bifurcation. The normal form on the center manifold for (1.4) of $E$ is given by (3.19), with $\lambda_{1}, \lambda_{2}, B_{1}, B_{2}$ defined by (3.20) and (3.21).

Copyright $@$ ( ) by SIAM. Unauthorized reproduction of this article is prohibited. 


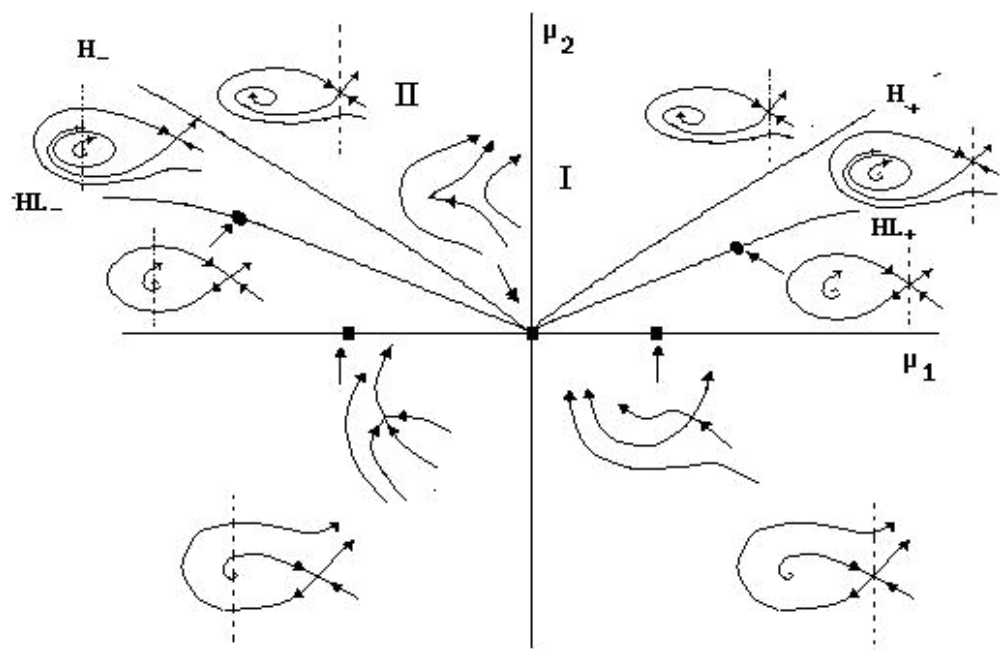

FIG. 2. The Bogdanov-Takens bifurcation diagram and phase portraits for system (3.22).

As an example, we consider system (1.4) with $r=1, K=2, A=1, D=\frac{1}{3}$; that is,

$$
\left\{\begin{array}{l}
\dot{x}(t)=x(t)\left(1-\frac{x(t)}{2}\right)-\frac{x(t) y(t)}{1+x(t)}, \\
\dot{y}(t)=y(t)\left(-\frac{1}{3}+\frac{x(t-\tau)}{1+x(t-\tau)}\right)-H .
\end{array}\right.
$$

According to Theorem 3.1, we know that system (3.22) has a Bogdanov-Takens singularity point $\left(x_{0}, y_{0}\right)=\left(\frac{5}{4}, \frac{27}{32}\right)$ when $\left(H_{0}, \tau_{0}\right)=\left(\frac{3}{16}, \frac{21}{10}\right)$. We will show that system (3.22) undergoes Bogdanov-Takens bifurcation when $\tau$ and $H$ vary in a small neighborhood of $\tau_{0}$ and $H_{0}$. Now we introduce two bifurcation parameters $\mu_{1}, \mu_{2}$ by setting $\tau=\frac{21}{10}+\mu_{1}, \beta_{1}=\frac{\alpha_{1} \beta_{2}}{\alpha_{2}}+\mu_{2}$; i.e., $H=H_{0}+\mu_{2}$. Following the analysis in this section, we obtain the versal unfolding for system (3.22) as follows:

$$
\left\{\begin{array}{l}
\dot{x}_{1}=x_{2}+\text { h.o.t. } \\
\dot{x}_{2}=\lambda_{1} x_{1}+\lambda_{2} x_{2}+B_{1} x_{1}^{2}+B_{2} x_{1} x_{2}+\text { h.o.t. }
\end{array}\right.
$$

where

$$
\begin{aligned}
& \lambda_{1}=-2.034602076 \mu_{2}, \quad \lambda_{2}=0.1614763552 \mu_{1}+1.919613031 \mu_{2}, \\
& B_{1}=0.3767781622, \quad B_{2}=-0.715752274 .
\end{aligned}
$$

Therefore, we know that system (3.22) exhibits Bogdanov-Takens bifurcation when the parameters $\mu_{1}, \mu_{2}$ vary in a small neighborhood of the origin. On the lines $H_{ \pm}$, there exists stable Hopf bifurcation, while there exists curves $H L_{ \pm}$corresponding to homoclinic bifurcation. The bifurcation diagram is depicted in Figure 2.

Remark 3.3. Similarly, we can study Bogdanov-Takens bifurcation in the following predator-prey model with predator harvesting and delayed prey specific growth

$$
\left\{\begin{array}{l}
\dot{x}(t)=r x(t)\left(1-\frac{x(t-\tau)}{K}\right)-\frac{x(t) y(t)}{A+x(t)}, \\
\dot{y}(t)=y(t)\left(-D+\frac{x(t)}{A+x(t)}\right)-H
\end{array}\right.
$$

and obtain results similar to Theorems 3.1 and 3.2. 
4. Discussion. Predator-prey models play a crucial role in studying the management of renewable resources (Clark [8]). The effect of constant-rate harvesting on the dynamics of predator-prey systems has been investigated by many authors; see, for example, Brauer and Soudack [3, 4, 5], Beddington and Cooke [1], Dai and Tang [11], Hogarth et al. [19], Myerscough et al. [30], Xiao and Jennings [35], and Xiao and Ruan [36]. Very rich and interesting dynamical behaviors, such as the existence of multiple equilibria, existence of Hopf bifurcation, limit cycles, homoclinic loops, and Bogdanov-Takens bifurcations, have been observed. It is also observed that in some cases, before a catastrophic harvest rate is reached the effect of harvesting is to stabilize the equilibrium of the population system.

Martin and Ruan [25] studied the combined effects of prey harvesting and delay on the dynamics of predator-prey systems and focused on three very well-studied delayed predator-prey models. Namely, they considered a generalized Gause-type predator-prey model with prey harvesting and a time delay in the prey specific growth term; a generalized Gause-type predator-prey model with prey harvesting and a time delay in the predator response function is analyzed; and the Wangersky-Cunningham predator-prey model with prey harvesting. It was shown that in the first and third models the time delay could cause not only instability and oscillations but also the switching of stabilities, while the prey harvesting changes only the equilibrium values but not the properties of solutions. In the second model, the time delay induces instability and bifurcation but there is no switching of stabilities; however, increasing the prey harvesting level will help the system to regain its stability. This indicates that the prey harvesting has a stabilizing effect on the dynamics of the model.

In this paper, following the work of Martin and Ruan [25] and Xiao and Ruan [36], we continued studying the combined effects of time delay and constant harvesting on the dynamics of predator-prey systems with Holling type II functional response. Two different types of models have been analyzed; namely, predator-prey systems with delayed predator response and (i) prey harvesting or (ii) predator harvesting. There are two types of bifurcation phenomena: Hopf bifurcation and Bogdanov-Takens bifurcation. More precisely, in the model with prey harvesting there is no bifurcation on the number of positive equilibria; time delay can induce oscillations of both species via Hopf bifurcation. While in the model with predator harvesting, multiple positive equilibria and degenerate equilibria can exist, Bogdanov-Takens bifurcation can occur.

In predator-prey interactions within fisheries systems, it is well known that the reduction of the predator stock level may increase the surplus production of the prey. Harvesting predators becomes controversial (May et al. [26], Flaaten [15], Yodzis [38]). The Bogdanov-Takens bifurcation diagram in the predator-prey models with predator harvesting in section 3 indicates that there are some parameter regions in which both predator and prey species can be driven to extinction. This may provide some explanations for the collapse of the Atlantic cod stocks in the Canadian Grand Banks (Hutchings and Myers [21], Myers et al. [27, 28], Hutchings [20]). Our study demonstrates that appropriate harvesting of predator population is crucial in the long-term survival of both predator and prey species, and in turn the fisheries systems. This is significant and useful in designing fishing policies for the fishery industry (Pauly et al. [31], Myers and Worm [29]).

Fishing is a seasonal activity. It will be very interesting to study how seasonal harvesting affects the existing Hopf and Bogdanov-Takens bifurcations in predator-prey systems with Holling type II functional response. We leave this for future consideration. 
Appendix A. In this section, we verify that $\alpha_{1} \beta_{2}-\alpha_{2} \beta_{1}=0$. Since

$$
\begin{aligned}
\alpha_{1} \beta_{2}-\alpha_{2} \beta_{1} & =\left[r-\frac{2 x_{0} r}{K}-\frac{A y_{0}}{\left(A+x_{0}\right)^{2}}\right]\left(-D+\frac{x_{0}}{A+x_{0}}\right)+\frac{A x_{0} y_{0}}{\left(A+x_{0}\right)^{3}} \\
& =r\left(1-\frac{2 x_{0}}{K}\right)\left(-D+\frac{x_{0}}{A+x_{0}}\right)+\frac{D A y_{0}}{\left(A+x_{0}\right)^{2}} \\
& =r\left(1-\frac{2 x_{0}}{K}\right) \frac{H}{y_{0}}+\frac{D A r\left(1-\frac{x_{0}}{K}\right)}{A+x_{0}} \\
& =\left(1-\frac{2 x_{0}}{K}\right) \frac{H}{\left(1-\frac{x_{0}}{K}\right)\left(A+x_{0}\right)}+\frac{D A r\left(1-\frac{x_{0}}{K}\right)}{A+x_{0}} \\
& =\frac{1}{A+x_{0}}\left[\frac{\left(1-\frac{2 x_{0}}{K}\right) H}{1-\frac{x_{0}}{K}}+D A r\left(1-\frac{x_{0}}{K}\right)\right] \\
& =\frac{1}{A+x_{0}}\left[\frac{K-2 x_{0}}{K-x_{0}} H+D A r\left(\frac{K-x_{0}}{K}\right)\right] \\
& =\frac{1}{A+x_{0}} \frac{K\left(K-2 x_{0}\right) H+D A r\left(K-x_{0}\right)^{2}}{K\left(K-x_{0}\right)},
\end{aligned}
$$

in order to prove $\alpha_{1} \beta_{2}-\alpha_{2} \beta_{1}=0$, we need only to prove $K\left(K-2 x_{0}\right) H+D A r(K-$ $\left.x_{0}\right)^{2}=0$. In fact,

$$
\begin{aligned}
K( & \left.-2 x_{0}\right) H+D A r\left(K-x_{0}\right)^{2} \\
& =K\left[K-2 \frac{K(1-D)+A D}{2(1-D)}\right] H+D A r\left[K-\frac{K(1-D)+A D}{2(1-D)}\right]^{2} \\
& =-\frac{A D K H}{1-D}+D A r\left[\frac{K}{2}-\frac{A D}{2(1-D)}\right]^{2} \\
& =\frac{-4 K A D H(1-D)+D A r[K(1-D)-A D]^{2}}{4(1-D)^{2}} \\
& =\frac{D A r K^{2}\left[\left(1-D-\frac{A D}{K}\right)^{2}-\frac{4 H(1-D)}{K r}\right]}{4(1-D)^{2}} \\
& =\frac{D A r K^{2}}{4(1-D)^{2}}\left[\left(1-D+\frac{A D}{K}\right)^{2}-\frac{4 A D r(1-D)+4 H(1-D)}{K r}\right] \\
& =\frac{D A r K^{2}}{4(1-D)^{2}}\left[\left(1-D+\frac{A D}{K}\right)^{2}-\frac{4(1-D)(A D r+H)}{K r}\right]=0 .
\end{aligned}
$$

Acknowledgments. We would like to thank Baochuan Tian for his help in plotting Figure 2. We are grateful to the referees for their careful reading, valuable comments, and helpful suggestions. Finally, we thank Teresa Faria for sending a copy of $[12]$.

\section{REFERENCES}

[1] J. R. Beddington and J. G. Cooke, Harvesting from a prey-predator complex, Ecol. Modelling, 14 (1982), pp. 155-177.

[2] F. Brauer, Stability of some population models with delay, Math. Biosci., 33 (1977), pp. 345358.

[3] F. Brauer And A. C. Soudack, Stability regions and transition phenomena for harvested predator-prey systems, J. Math. Biol., 7 (1979), pp. 319-337.

Copyright $@$ by SIAM. Unauthorized reproduction of this article is prohibited. 
[4] F. Brauer And A. C. Soudack, Stability regions in predator-prey systems with constant-rate prey harvesting, J. Math. Biol., 8 (1979), pp. 55-71.

[5] F. Brauer And A. C. Soudack, Coexistence properties of some predator-prey systems under constant rate harvesting and stocking, J. Math. Biol., 12 (1981), pp. 101-114.

[6] J. M. Casey And R. A. Myers, Near extinction of a large widely distributed fish, Science, 226 (1998), pp. 690-692.

[7] S. N. Chow And J. K. Hale, Methods of Bifurcation Theory, Springer-Verlag, New York, 1982.

[8] C. W. Clark, Mathematical Bioeconomics, The Optimal Management of Renewable Resources, 2nd ed., John Wiley \& Sons, New York-Toronto, 1990.

[9] K. L. Cooke and Z. Grossman, Discrete delay, distributed delay and stability switches, J. Math. Anal. Appl., 86 (1982), pp. 592-627.

[10] J. M. Cushing, Integrodifferential Equations and Delay Models in Population Dynamics, Springer-Verlag, Heidelberg, 1977.

[11] G. DAI AND M. TANG, Coexistence region and global dynamics of a harvested predator-prey system, SIAM J. Appl. Math., 58 (1998), pp. 193-210.

[12] T. FARIA, On the study of singularities for a planar system with two delays, Dyn. Contin., Discrete Impuls. Syst., 10 (2003), pp. 357-371.

[13] T. FARIA And L. T. Magalhães, Normal forms for retarded functional differential equations with parameters and applications to Hopf bifurcation, J. Differential Equations, 122 (1995), pp. 181-200.

[14] T. FARIA And L. T. Magalhães, Normal forms for retarded functional differential equations and applications to Bogdanov-Takens singularity, J. Differential Equations, 122 (1995), pp. 201-224.

[15] O. FlaAten, The Economics of Multispecies Harvesting-Theory and Application to the Barants Sea Fisheries, Springer, Berlin, 1988.

[16] O. FlaAten, On the bioeconomics of predator-prey fishing, Fish. Research, 37 (1998), pp. 179191.

[17] K. Gopalsamy, Stability and Oscillations in Delay Differential Equations of Population Dynamics, Kluwer Academic, Dordrecht, 1992.

[18] J. K. Hale, Theory of Functional Differential Equations, Springer-Verlag, New York, 1977.

[19] W. L. Hogarth, J. Norbury, I. Cunning, and K. Sommers, Stability of a predator-prey model with harvesting, Ecol. Modelling, 62 (1992), pp. 83-106.

[20] J. A. Hutchings, Collapse and recovery of marine fishes, Nature, 406 (2000), pp. 882-885.

[21] J. A. Hutchings And R. A. Myers, What can be learned from the collapse of a renewable resource? Atlantic code, Gadus morhua, of Newfoundland and Labrador, Can. J. Fish. Aquat. Sci., 51 (1994), pp. 2126-2146.

[22] J. B. C. JACKSON ET AL., Historical overfishing and the recent collapse of coastal ecosystems, Science, 293 (2001), pp. 629-638.

[23] Y. Kuang, Delay Differential Equations with Applications in Population Dynamics, Academic Press, New York, 1993.

[24] Y. A. Kuznetsov, Elements of Applied Bifurcation Theory, Springer-Verlag, New York, 1995.

[25] A. Martin and S. Ruan, Predator-prey models with delay and prey harvesting, J. Math. Biol., 43 (2001), pp. 247-267.

[26] R. May, J. R. Beddington, C. W. Clark, S. J. Holt, and R. M. Laws, Management of multispecies fisheries, Science, 205 (1979), pp. 267-277.

[27] R. A. Myers, N. J. Barrowman, J. A. Hutchings, and A. A. Rosenberg, Population dynamics of exploited fish stocks at low population level, Science, 269 (1995), pp. 11061108.

[28] R. A. Myers, J. A. Hutchings, and N. J. Barrowman, Why do fish stocks collapse? The example of cod in Atlantic Canada, Ecol. Appl., 7 (1997), pp. 91-106.

[29] R. A. Myers and B. Worm, Rapid worldwide depletion of large predatory fish communities, Nature, 423 (2003), pp. 280-283.

[30] M. R. Myerscough, B. E. Gray, W. L. Hograth, and J. Norbury, An analysis of an ordinary differential equation model for a two-species predator-prey system with harvesting and stocking, J. Math. Biol., 30 (1992), pp. 389-411.

[31] D. Pauly Et Al., Towards sustainability in world fisheries, Nature, 418 (2002), pp. 689-695.

[32] S. RuAn, Absolute stability, conditional stability and bifurcation in Kolmogorov-type predatorprey systems with discrete delays, Quart. Appl. Math., LIX (2001), pp. 159-173.

[33] S. RuAn, On nonlinear dynamics of predator-prey models with discrete delay, Math. Model. Natural Phenom., 4 (2009), pp. 140-188.

[34] Q. Schiermeier, How many more fish in the sea?, Nature, 419 (2002), pp. 662-665.

Copyright (c) by SIAM. Unauthorized reproduction of this article is prohibited. 
[35] D. Xiao And L. S. Jennings, Bifurcations of a ratio-dependent predator-prey system with constant rate harvesting, SIAM J. Appl. Math., 65 (2005), pp. 737-753.

[36] D. XiaO And S. Ruan, Bogdanov-Takens bifurcations in predator-prey systems with constant rate harvesting, Fields Inst. Commun., 21 (1999), pp. 493-506.

[37] D. XIAo And S. Ruan, Multiple bifurcations in a delayed predator-prey system with nonmonotonic functional response, J. Differential Equations, 176 (2001), pp. 494-510.

[38] P. YodzIS, Predator-prey theory and management of multispecies fisheries, Ecol. Appl., 4 (1994), pp. 51-58.

Copyright (c) by SIAM. Unauthorized reproduction of this article is prohibited. 